\title{
Theory of the spiral core in excitable media
}

\author{
David A. Kessler ${ }^{a}, 1$, Herbert Levine ${ }^{b}$ and William N. Reynolds ${ }^{b}$ \\ ${ }^{a}$ Department of Physics, Univ. of Michigan, Ann Arbor, MI 48109, USA \\ 'Department of Physics and Institute for Nonlinear Science, University of California, \\ San Diego, La Jolla, CA 92093, USA
}

Received 8 March 1993

Revised manuscript received 15 July 1993

Accepted 16 July 1993

Communicated by $\mathrm{H}$. Flaschka

\begin{abstract}
A rigorous asymptotic spiral solution to an excitable reaction diffusion system is found by separating space into two scaling regions: an "outer region", having the same spatial scaling as the overall spiral structure, which exhibits a singularity at the spiral tip; and a "core" region around the spiral tip, where space is scaled so as to resolve the tip singularity. The stability of the spiral structure is investigated for both the outer region, which is found to possess no intrinsic instability, and the core region which is found to be unstable. Both the zero and small diffusion systems are found to exhibit qualitatively similar behaviour. The implication of these results for both experiment and simulation, in particular within the context of the observed "meandering" instability, are addressed.
\end{abstract}

\section{Introduction}

Excitability and spiral formation are phenomena that are found in many physical and biological systems. Due to the ubiquity of excitable behaviour, there have been many different models of excitability in various physical and biological systems proposed over the years. Some of the most notable examples include the Lotka-Volterra predator-prey model and its many extensions [1], the Hodgkin-Huxley model of signal propagation in the giant squid axon and its simplification, the Fitzhugh-Nagumo model $[2,3]$, the Oregonator model of the BelusovZhabotinskii reaction [4], the Martiel-Goldbeter model of signal generation in Dictyostelium Discodeum [5] and the Maple-Turner-Sales model

\footnotetext{
${ }^{1}$ Permanent Address: Dept. of Physics, Bar-Ilan Univ., Ramat Gan, Israel.
}

of catalysis of $\mathrm{CO}$ on $\mathrm{Pt}$ substrates [6]. The remarkable thing about all of these models, representing as they do, such a wide variety of systems, is that they share the generic behaviour of spiral formation, selection and stability, independent of the details of the particular system.

Over the past decade, spiral formation in excitable media has been the focus of a considerable body of work [7]. As a result, spiral formation has been fairly well characterized. It has been demonstrated that much of the behaviour of excitable media can be reproduced with greatly simplified models of real systems. Two of the most successful models of this type are the two variable Oregonator model and the two variable Fitzhugh-Nagumo model. Both of these models consider the dynamics of two fields with greatly differing temporal scales - which, accordingly, are referred to as the "fast" and "slow" fields. Studies of these models have shown that for a given parameter set, spirals select a unique 
rotation frequency and shape. Both spiral selection and plane wave dispersion, which have been observed in these simplified theoretical models, have also been observed experimentally, particularly in experiments on the BelusovZhabotinskii reaction [8]. It has become clear that, for the case of greatly differing reaction rates, these phenomena are due to joint effects of the dispersiveness of the media and the "Gibbs-Thomson" effect [9] which causes a decrease in interfacial velocity in the presence of interfacial curvature.

Keener [10], and later Keener and Tyson [11], used this conjecture to develop a geometric model of the spiral interface, which couples the dispersive dynamics of planar periodic structures, characteristic of the kinetics of the media, to a differential expression of the aforementioned Gibbs-Thomson relation. In this way, they were able to relate the rotation frequency of the spiral to the tip rotation radius. Although this analysis stimulated much later work, it did not fully address the issue of frequency selection, nor was it possible to investigate the question of spiral stability. Moreover, more rigorous investigations have brought into question the validity of this model's results, as it depends on the assumption of thin, non-interacting interfaces, which is invalid near the spiral tip where length scales are comparable to those of the interfacial structure $^{\# 1}$. Similarly, work by Pelce and Sun [13] did not include a completely consistent theory of the core. This was pointed out by Keener [14] who provided a possible resolution of this problem for the special case of symmetric spirals. Also, Karma [15] showed that the PelceSun method could be used to find a solution for the weakly-excitable limit.

Recently, progress has been made in formulating a more rigorous description of spiral waves in certain models of excitable media. Solutions have been found for media having a vanishing

\footnotetext{
*1 The same remarks hold for the kinematic approach to spirals used by the Russian school [12].
}

reaction rate ratio and either small (a case first considered by Bernoff [16]) or zero $[17,18]$ diffusion in the core. For these cases, the size of the core region is much smaller than the asymptotic wavelength of the spiral. One can then show that a rescaling of space in the vicinity of the core and matching to the previously determined far-field spiral allows for a consistent solution everywhere in space. As a result, it is now possible to calculate the linear stability of spiral structures, and to compare the results to numerical and experimental studies of spiral formation.

Another phenomenon that has been observed in theoretical models of excitable media $[19,22,20]$, and experimentally [8], is the transition to tip meandering. In this transition, the spiral tip, which rotates uniformly for media where the ratio $\epsilon^{-1}$ of the fast and slow fields' reaction rates is small enough, exhibits, as this ratio is increased, a second frequency that is almost commensurate with the rotation frequency, resulting in meandering, "flower"-like trajectories of the rotating spiral tip. Recent advances in numerical simulations by Barkley, Karma and Winfree [20-22] have emnabled a rather complete description of these transitions, allowing a mapping of the phase diagram of the various behaviours in parameter space. This transition has been shown computationally to be a Hopf bifurcation. Both the spiral's linear stability and the eigenmodes of the linearized stability operator have been calculated numerically by Barkley [23], providing useful checks and impetus for analytic work. This work has also suggested that these behaviours are independent of the details of the model used and of the magnitude of the slow field's diffusion. Indeed, it appears that the instability is qualitatively similar for the case of fields with equal diffusion and for the case of zero diffusion of the slow field. More recently, the phenomenon of "hypermeander" has been observed [22]. Here, the motion of the tip is more complex than the two frequency motion observed in simple mean- 
dering and the tip traces out an irregular, perhaps chaotic, path. The precise nature of the hypermeander remains an open area of research.

In this work, we first review briefly the formulation of a rigorous asymptotic solution to the spiral formation problem for both small and zero diffusion in the slow field, in the limit of vanishing reaction rate ratio, demonstrating that the competition between dispersion and curvature lead us to an eigenvalue problem for the spiral interface and the rotation frequency. The spiral core is described in both cases by a rescaled set of equations valid in a small region around the spiral's center. We then calculate the linear stability of both the spiral and its core for each case, concluding that in this limit, the spiral core is unstable for both systems, while in the outer region the spiral itself is stable. We thus interpret the meandering instability as a localized core instability acting as a source for waves which then propagate out along the spiral. Moreover, we show that this instability is independent of the details of the model used, and should be observed in any two variable system capable of supporting excitable waves. Some of our results, in preliminary form, have appeared elsewhere [24].

It should be pointed out that our work leaves unresolved one important feature of the mean- dering instability. As already stated, simulation has shown that the transition to meandering is a Hopf bifurcation; our unstable mode, in the small $\epsilon$ limit, has a purely real growth rate. This is not necessarily a disagreement, since none of the simulations can be performed in the limit accessible to asymptotic analysis. In the concluding section, we discuss possible efforts for bridging the gap between simulations and asymptotics, by remedying the unphysical decoupling of the core and the outer solution which occurs to leading order in $\epsilon^{1 / 3}$.

\section{Background}

In this work we will be considering a two variable model of an excitable media given by the reaction-diffusion system

$$
\dot{u}=\epsilon \nabla^{2} u+\frac{f(u, v)}{\epsilon}, \quad \dot{v}=\delta \epsilon \nabla^{2} v+g(u, v) .
$$

For what we will refer to as the "diffusionless" case, $\delta=0$, and for the "diffusive" case (the Bernoff limit), $\delta \ll 1$. Generically, $f$ and $g$ have nullclines as in fig. 1 . Since the dynamics of $u$ are $\mathcal{O}(1 / \epsilon)$ faster than those of $v$, we refer to $u$ and $v$ as the "fast" and "slow" fields respectively. As a specific example of an excitable system, we will

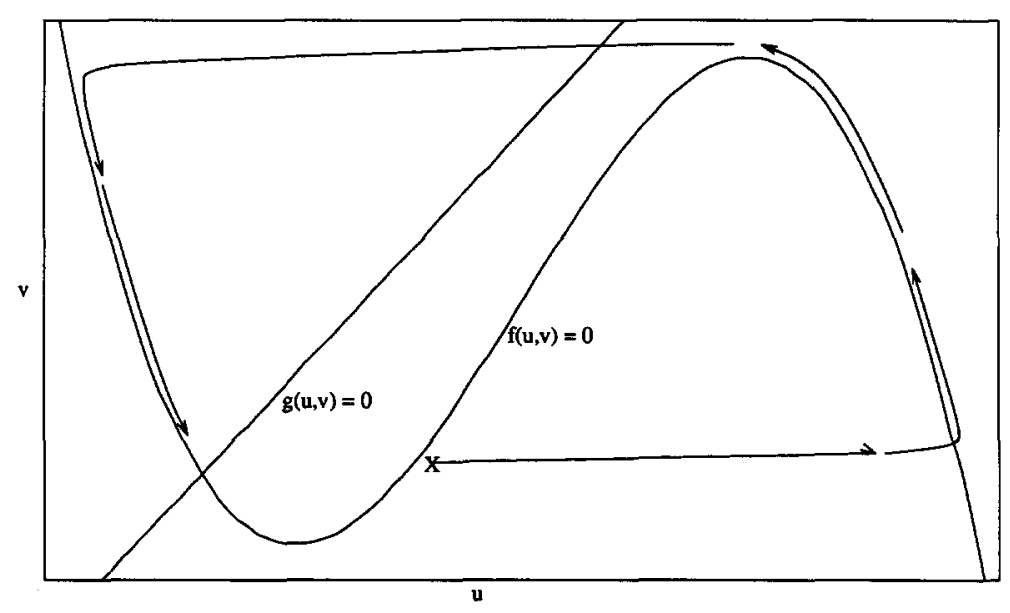

Fig. 1. The nullclines $f(u, v)=g(u, v)=0$, and a trajectory in phase space. 
use the Fitzhugh-Nagumo (FN) model [3]. The kinetics of this model are given by

$f(u, v)=3 u-u^{3}-v$,

$g(u, v)=u-\gamma v+\Delta$.

We will first review the standard analysis of periodic pulses in $1 \mathrm{D}$ excitable media $[11,25]$. We move to a stretched travelling coordinate system, $z=(x-c t) / \epsilon$. Under this transformation, the system (1) becomes

$\frac{\partial^{2} u}{\partial z^{2}}+c \frac{\partial u}{\partial z}+f(u, v)=0$,

$\delta \frac{\partial^{2} v}{\partial z^{2}}+c \frac{\partial v}{\partial z}+\epsilon g(u, v)=0$.

To lowest order in $\epsilon$, we see that $v$ is indeed a constant, $v=v_{\mathrm{I}}$. We look for a domain wall solution with boundary conditions such that as $z \rightarrow \infty, u \rightarrow u^{-}\left(v_{1}\right)$, and as $z \rightarrow-\infty, u \rightarrow u^{+}\left(v_{1}\right)$. This choice of boundary conditions implicitly leads to the convention that a front where the quiescent phase moves into the excited phase has negative velocity and one with the excited phase moving into the quiescent phase has positive velocity. The system (4) is a nonlinear eigenvalue problem for $c\left(v_{1}\right)$, which can be solved numerically. It is readily seen that there exists a "stall concentration", $v_{\mathrm{s}}$, when $\int_{u^{-}\left(v_{\mathrm{s}}\right)}^{u^{+}\left(v^{\prime}\right)} f\left(u, v_{\mathrm{s}}\right) \mathrm{d} u$ $=0$. For the FN model (3), $v_{\mathrm{s}}=0$. A plot of $c\left(v_{1}\right)$ for the $\mathrm{FN}$ model is given in fig. 2.

One can easily construct a periodic train of excited and quiescent zones separated by alternating positive and negative-velocity interfaces. This is done by integrating the slow field equation, using the steady train boundary conditions, $c\left(v_{\mathrm{b}}\right)=-c\left(v_{\mathrm{f}}\right)=c$. To zeroth order in $\epsilon$ and $\delta$, the dispersion relation between period and velocity is given by

$T=\int_{v_{\mathrm{f}}}^{v_{\mathrm{b}}} \frac{\mathrm{d} v}{g\left(u^{+}(v), v\right)}+\int_{v_{\mathrm{b}}}^{v_{\mathrm{f}}} \frac{\mathrm{d} v}{g\left(u^{-}(v), v\right)}$.

Let us now turn to two dimensions. As is

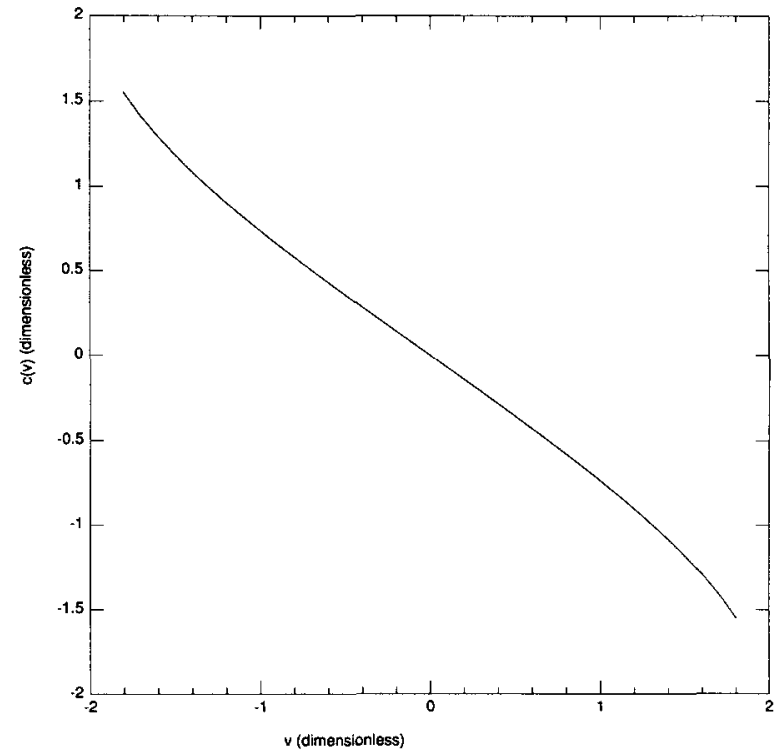

Fig. 2. Plot of the wavefront velocity, $c$, vs the slow field concentration in the front, $v$.

shown in $[10,11,25]$, the Laplacian operator in this coordinate system is given by

$\nabla^{2}=\frac{\partial^{2}}{\partial \lambda^{2}}+\epsilon \kappa(s) \frac{\partial}{\partial \lambda}+\mathcal{O}\left(\epsilon^{2}\right)$,

where $\kappa$ is the curvature of the interface, and $\lambda$ is the derivative along the interface normal. If we assume that the interface is moving steadily with velocity $c_{n}$, the time derivative is given by

$\frac{\partial}{\partial t}=-c_{n}(s) \frac{\partial}{\partial \lambda}$,

where we label the curve by the arclength $s$. In this coordinate system, our system (1) becomes

$\frac{\partial^{2} u}{\partial \lambda^{2}}+\left[c_{n}(s)+\epsilon \kappa(s)\right] \frac{\partial u}{\partial \lambda}+f(u, v)=\mathscr{O}\left(\epsilon^{2}\right)$.

Comparing this to (4) we see that the interface velocity is related to the value of the $v$ field on the interface by

$c_{n}\left(r_{\mathrm{I}}\right)=c\left(v\left(\boldsymbol{r}_{\mathrm{I}}\right)\right)-\epsilon \kappa\left(\boldsymbol{r}_{\mathrm{I}}\right)$,

where $r_{I}$ is the position of the interface. This is analogous to the well known Gibbs-Thomson 
(with kinetic coefficient) relationship for interfacial velocity in solidification [26].

The fact that $\epsilon$ is vanishingly small might lead one to believe that the curvature term in (9) can be dropped. Fife [27] suggested the alternate hypothesis that the curvature term must be included so as to give a meaningful theory. This led him to postulate that spiral formation will occur on spatial and temporal scales such that all the terms of (9) are of equal magnitude. Note that this is not the only logical possibility; dendritic crystals choose their growth velocity in such a way as to make the curvature contribution vanishingly small for the actual solution, even though one must include this term to get a physically meaningful theory [9].

In this Fife scaling, $v-v_{\mathrm{s}}=\epsilon^{1 / 3} \tilde{v}, x=\epsilon^{2 / 3} \tilde{x}$ and $t=\epsilon^{1 / 3} \tilde{t}$. Under this scaling, $c\left(v_{\mathrm{I}}\right) \simeq \epsilon^{1 / 3} c_{v} \tilde{v}_{\mathrm{I}}$, where $c_{v}=\left.[\mathrm{d} c(v) / \mathrm{d} v]\right|_{v=0}$ and the interfacial equation (9) becomes (after dropping the tildes)

$c_{n}\left(\boldsymbol{r}_{\mathrm{I}}\right)=c_{v} v\left(\boldsymbol{r}_{\mathrm{I}}\right)-\kappa\left(\boldsymbol{r}_{\mathrm{I}}\right)$.

The equation of motion for the $v$ field, (1), simplifies tremendously under the Fife ansatz. In particular, whereas in general $g(u, v)=g\left(u^{ \pm}(v)\right.$, $v$ ) is a complicated nonlinear function of $v$, in the Fife ansatz $v \approx v_{s}$ everywhere, so that $g(u, v) \approx$ $g\left(u^{ \pm}\left(v_{s}\right), v_{s}\right) \equiv g^{ \pm}$, is (a different) constant in both the excited and quiescent regions. The remaining field equation to lowest order in $\epsilon$ is then, after Fife rescaling and dropping of the tildes,

$\delta \nabla^{2} v+g^{ \pm}=\frac{\partial v}{\partial t}$.

This, in conjunction with the Fife-rescaled interfacial equation (10), gives a free-boundary problem for the shape of the interface and the fields $u(\boldsymbol{r})$ and $v(\boldsymbol{r})$.

Finally, we follow the work of Bernoff [16] and Karma [17], to obtain a steadily rotating spiral solution to the system $(10,11)$ in the small $\delta$ limit. In this limit, we may drop the $\delta$ term in (11) except in a small region around the origin. Transforming to the frame corotating with the spiral at the large angular frequency $\omega \equiv \epsilon^{-1 / 3} \tilde{\omega}$, (10) and (11) become

$c_{n}\left(\boldsymbol{r}_{\mathrm{I}}\right)=c_{v} v\left(\boldsymbol{r}_{\mathrm{I}}\right)-\kappa\left(\boldsymbol{r}_{\mathrm{I}}\right)$,

$\omega \frac{\partial v}{\partial \theta}+g^{ \pm}=0$.

To solve this system, we first integrate the second equation obtaining

$v^{ \pm}(r, \theta)=-g^{ \pm} \theta / \omega+b^{ \pm}(r)$,

where $b^{ \pm}$are constants of integration. The excited and quiescent regions will be separated by two interfaces, $\theta^{ \pm}(r)$, whose shapes will be asymptotic to Archimedean spirals, $\theta(r) \sim r$. We determine the angular width of the excited zone, $\Delta \theta(r) \equiv \theta^{+}(r)-\theta^{-}(r)$ by imposing continuity at the interfaces, $v^{+}\left(\theta^{+}(r)\right)=v^{-}\left(\theta^{+}(r)\right)$ and $v^{+}\left(\theta^{-}(r)\right)=v^{-}\left(2 \pi+\theta^{-}(r)\right)$. This yields

$\Delta \theta=\frac{2 \pi g^{-}}{g^{-}-g^{+}}$,

which is independent of $r$. We see that the spiral consists of two interfaces differing only by a constant angular shift. Since the spiral is a steady structure, $c_{n}\left[\theta^{+}(r)\right]=-c_{n}\left[\theta^{-}(r)\right]$. The symmetry of $\theta^{+}(r)$ and $\theta^{-}(r)$ imply that $\kappa\left(\theta^{+}\right)=-\kappa\left(\theta^{-}\right)$, so we are left with the condition $v_{\mathrm{I}}\left(\theta^{+}(r)\right)=$ $-v_{\mathrm{I}}\left(\theta^{-}(r)\right)$. This, together with (13) leads to the condition that $v_{\mathrm{I}}$ on the front and back interfaces are constants independent of $r$, given by

$v_{\mathrm{I}}\left(\theta^{ \pm}(r)\right) \equiv v_{\mathrm{I}}^{ \pm}= \pm \frac{\left(g^{+} g^{-}\right) \pi}{\omega\left(g^{+}-g^{-}\right)}$.

As the two interfaces come together at the spiral tip, the $v$ field has a discontinuity, and the interface is cusped. This unphysical behaviour can be resolved by rescaling space near $r=0$, and will be addressed in section 3 .

To determine the shape of the spiral, we write down the curvature and normal velocity operators for an interface parametrized by $\theta_{+}(r)$ :

$\kappa=-\frac{1}{\left(1+\psi^{2}\right)^{3 / 2}} \frac{\mathrm{d} \psi}{\mathrm{d} r}-\frac{\psi}{r \sqrt{1+\psi^{2}}}$, 
$c_{n}=\frac{\omega r}{\sqrt{1+\psi^{2}}}$,

where $\psi(r) \equiv r\left(\mathrm{~d} \theta_{+} / \mathrm{d} r\right)$. Inserting these relations into the first equation of (12), using (15), and making the rescaling $\rho=\sqrt{\omega} r$, we arrive at the following nonlinear ordinary differential equation for the interface:

$\frac{\mathrm{d} \psi}{\mathrm{d} \rho}=\left(\rho-\frac{\psi}{\rho}\right)\left(1+\psi^{2}\right)-B\left(1+\psi^{2}\right)^{3 / 2}$,

where $B=c_{v} v_{\mathrm{I}}^{+} / \sqrt{\omega}=c_{v} g^{+} g^{-} \pi / \omega^{3 / 2}\left(g^{+}-g^{-}\right)$. For the FN model, $g^{ \pm}= \pm \sqrt{3}+\Delta, c_{v}=-1 / \sqrt{2}$ and so $B=\pi\left(3-\Delta^{2}\right) / 2 \sqrt{6} \omega^{3 / 2}$. Spirals will be those solutions which satisfy the boundary conditions $\psi(\rho) \sim-\rho$ as $\rho \rightarrow \infty$ and $\psi(\rho) \sim \rho$ as $\rho \rightarrow 0$. These solutions can be calculated numerically by shooting. The selected eigenvalue, $B \doteq 1.738$, determines the spiral's rotation frequency, which in Fife-scaled units, is

$\omega^{*}=\left(\frac{c_{v} g^{+} g^{-} \pi}{B\left(g^{+}-g^{-}\right)}\right)^{2 / 3}$.

A plot of the solution, $\theta^{+}(\rho)=\int(\psi / \rho) \mathrm{d} \rho$ is given in fig. 3 . This equation and the resulting quantization condition for the frequency appeared first in the work of Keener and Tyson

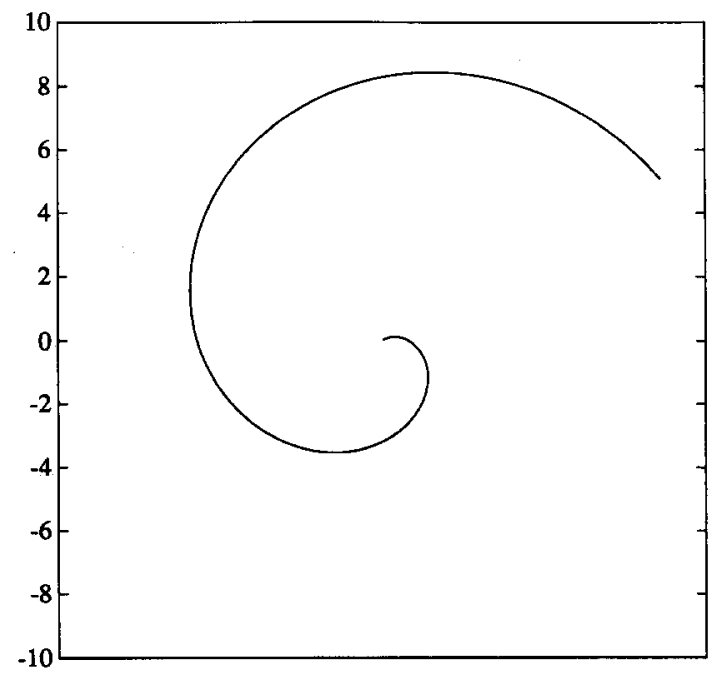

Fig. 3. Spiral solution to (18), plotted in $x-y$ space.
[11]; (actually in a different context this equation was first written down by Burton, Cabrera and Frank [28]). However, it was not clearly stated in this work that this equation is only (asymptotically) exact for the case of small or zero $\delta$.

\section{Core solutions}

As previously mentioned, the spiral solution in the outer regions exhibits a singularity as $r \rightarrow 0$. This problem can be resolved by rescaling the system (1) near $r=0$ and asymptotically matching the solutions of the resulting equations to the outer region solution just described. Since the term $\delta \nabla^{2} v$ in (1) is a singular perturbation of the system, the rescaling we use will be determined by whether $0<\delta \ll 1$ or $\delta=0$.

\subsection{The diffusionless core}

Singularities develop at the origin in the outer solution because the assumptions made during its derivation break down near this point. In the diffusionless case [18], the assumption that the width of the excited and quiescent regions (which scale as $r \Delta \theta$ ), is much greater than the width of the reaction zones (which scale as $\epsilon$ ) breaks down as $r \rightarrow \epsilon$; here, all the fields are changing over a spatial scale of $\epsilon$. This suggests that we should introduce the new scaling $\hat{r}=\epsilon r$ in (1). The frequency $\omega$ is that given by the outer solution, $\omega=\epsilon^{-1 / 3} \omega^{*}$. We also expand $u$ and $v$ in power series in $\epsilon^{1 / 3}$ :

$u=u_{0}+\epsilon^{1 / 3} u_{1}+\mathcal{O}\left(\epsilon^{2 / 3}\right)$,

$v=v_{0}+\epsilon^{1 / 3} v_{1}+\mathcal{O}\left(\epsilon^{2 / 3}\right)$.

At zeroth order, this leads to the system

$\nabla^{2} u_{0}+f\left(u_{0}, v_{0}\right)=0, \quad \omega^{*} \frac{\partial v_{0}}{\partial \theta}=0$,

which immediately yields the fact that $v_{0}$ is only a function of $r$. At first order, 


$$
\begin{aligned}
& \nabla^{2} u_{1}+\left.\frac{\partial f}{\partial u}\right|_{u_{0}, v_{0}} u_{1}+\left.\frac{\partial f}{\partial v}\right|_{u_{0}, v_{0}} v_{1}=0 \\
& \omega^{*} \frac{\partial v_{1}}{\partial \theta}=g\left(u_{0}, v_{0}\right) .
\end{aligned}
$$

A solvability condition arises in that $v_{1}$ must be single valued in $\theta$. This implies that

$$
\int_{0}^{2 \pi} g\left(u_{0}, v_{0}\right) \mathrm{d} \theta=0 \text {. }
$$

This equation, valid for all $r$, implicitly determines $v_{0}(r)$ as a functional of $u_{0}(r, \theta)$.

In the outer region, $v \sim \mathscr{O}\left(\epsilon^{1 / 3}\right)$ and $u=u^{ \pm}$, where $f\left(u^{ \pm}, 0\right)=0$. This leads to the boundary conditions

$$
\begin{aligned}
& \lim _{r \rightarrow \infty} u_{0}(r, \theta)=\left\{\begin{array}{ll}
u^{+} & 0 \leq \theta \leq \Delta \theta, \\
u^{-} & \Delta \theta<\theta<2 \pi
\end{array},\right. \\
& \lim _{r \rightarrow \infty} v_{0}(r, \theta)=0,
\end{aligned}
$$

where we have in addition fixed the rotational phase of the spiral. The system (21), (23), (24) determines the solution in the core region for the case of diffusionless media.

As an example, we consider the FN dynamics. In this case, (23) becomes

$$
\int_{0}^{2 \pi}\left[u_{0}(r, \theta)-\gamma v_{0}(r)+\Delta\right] \mathrm{d} \theta=0 .
$$

This in turn implies

$v_{0}(r)=\frac{1}{2 \pi \gamma} \int_{0}^{2 \pi}\left[u_{0}(r, \theta)+\Delta\right] \mathrm{d} \theta$

Evaluating both sides at $r \rightarrow \infty$ by use of (24) leads to the relation

$\Delta \theta u^{+}+(2 \pi-\Delta \theta) u^{-}+\Delta=0$,

which gives the same value $\Delta \theta=2 \pi\left(u^{-}+\Delta\right) /$ $\left(u^{-}-u^{+}\right)$as we determined for the outer region, as it must.

In the general case, the system (21), (23), (24) can be thought as the minimization of the Lyapunov functional

$$
\int \mathrm{d}^{2} x\left[\left(\nabla u_{0}\right)^{2}+F\left(u_{0}, v_{0}\right)\right]
$$

where $f(u, v)=\partial F(u, v) / \partial u$. In this formulation, the condition (23) plays the role of a constraint.

Solutions to (21), (23), (24) were generated numerically using a simple relaxational solver. $u_{0}(r)$ was first discretized over a $100 \times 100$ rectangular grid of size $R$ and grid spacing $\Delta x=$ $\frac{1}{100} R$. $R$ was typically $12.5,15.0$ or 25.0 . To do an update of the $u_{0}$ field it was necessary to calculate $v_{0}(r)$. This was done using (26), employing a spline interpolation of the $u_{0}$ field. The integral at radius $r$ was performed via the trapezoidal rule, with $2 \pi r / \Delta x$ points. The $v_{0}$ field was calculated for 50 evenly spaced points in the interval $[0, R]$ and $v_{0}(r)$ determined by cubic spline interpolation.

Solutions were actually calculated on a circular subdomain of radius $R_{\mathrm{m}}=0.8 R$ (40 lattice spacings). Once $v_{0}$ is calculated for a given site, an updated value of $u_{0}$ could also be calculated. This was done using the differencing scheme, given by

$$
\begin{aligned}
u_{t+1}= & {\left[u_{t}+\Delta t\left(L\left[u_{t}\right]+f\left(u_{t}, v_{t}\right)-\frac{\partial f\left(u_{t}, v_{t}\right)}{\partial u} u_{t}\right)\right] } \\
& /\left(1-\Delta t \frac{\partial f\left(u_{t}, v_{t}\right)}{\partial u}\right)
\end{aligned}
$$

Here $L\left[u_{t}\right]$ is a discretized version of the Laplacian, which, for this simulation, was a five point formula:

$$
\begin{aligned}
L\left[u_{t}\left(x_{n}, y_{n}\right)\right]= & \frac{1}{(\Delta x)^{2}}\left[u_{t}\left(x_{n+1}, y_{n}\right)\right. \\
& +u_{t}\left(x_{n-1}, y_{n}\right)+u_{t}\left(x_{n}, y_{n+1}\right) \\
& \left.+u_{t}\left(x_{n}, y_{n-1}\right)-4 u_{t}\left(x_{n}, y_{n}\right)\right] .
\end{aligned}
$$

The lattice was initialized by setting $u_{0}=u^{+}$ within a sector of angular width $\Delta \theta$, and $u_{0}=u^{-}$ everywhere else. Note that this implicitly sets the boundary condition to be $u_{0}=u^{ \pm}$(depending on 

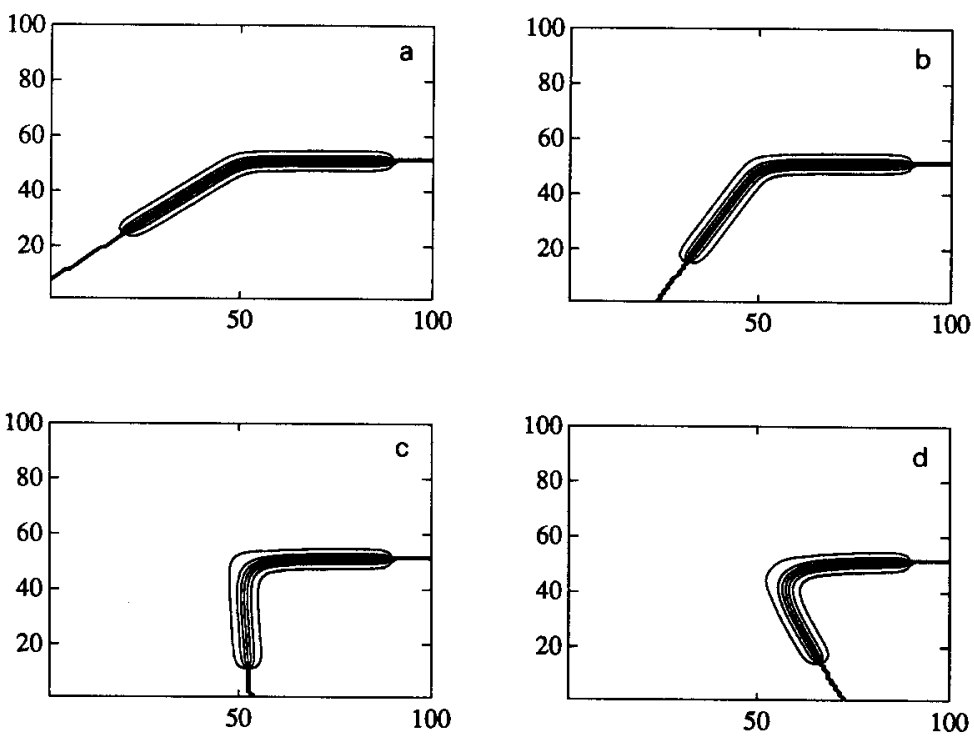

Fig. 4. Contour plots of tip solutions for the diffusionless case. Plots (a), (b), (c), (d) are for $\Delta=0.4,0.6,0.9$ and 1.1 .

the angular position of site) on the circumference of the subdomain, since updates were not done exterior to it. In fig. 4 , we plot these numerical solutions for several values of $\Delta$.

We can predict some of the solution's qualitative features. For $\Delta=0,(21),(23),(24)$ reduces to a $1 \mathrm{D}$ problem, with $v_{0}(r)=0$ and $u_{0}(x$, $y)=\sqrt{3} \tanh \left(\sqrt{\frac{3}{2}} x\right)$. Note that for this solution, the "interface", which may be associated with the contour line $u_{0}(r)=0$, is a straight line which passes through the origin. As we increase $\Delta$ away from zero, two interfaces come together in a tip, breaking the translational symmetry in $y$. The Laplacian term in (21) "smooths out" this tip, causing it to recede from the origin, and the core radius $r_{\mathrm{c}}$ (the point of closest approach of the contour line $u_{0}(\boldsymbol{r})=0$ to the origin) to increase. As $\Delta$ is increased, the tip becomes sharper and this "melting back" effect more pronounced. In fig. 5 we plot $r_{\mathrm{c}}$ as function of $\Delta$ for several different domain sizes. $r_{\mathrm{c}}$ is an increasing function of $\Delta$, independent of the domain size for reasonable values of $\Delta$. For larger $\Delta, r_{c}$ approaches the domain size and boundary effects become important, making impractical our numerical procedure in this limit.

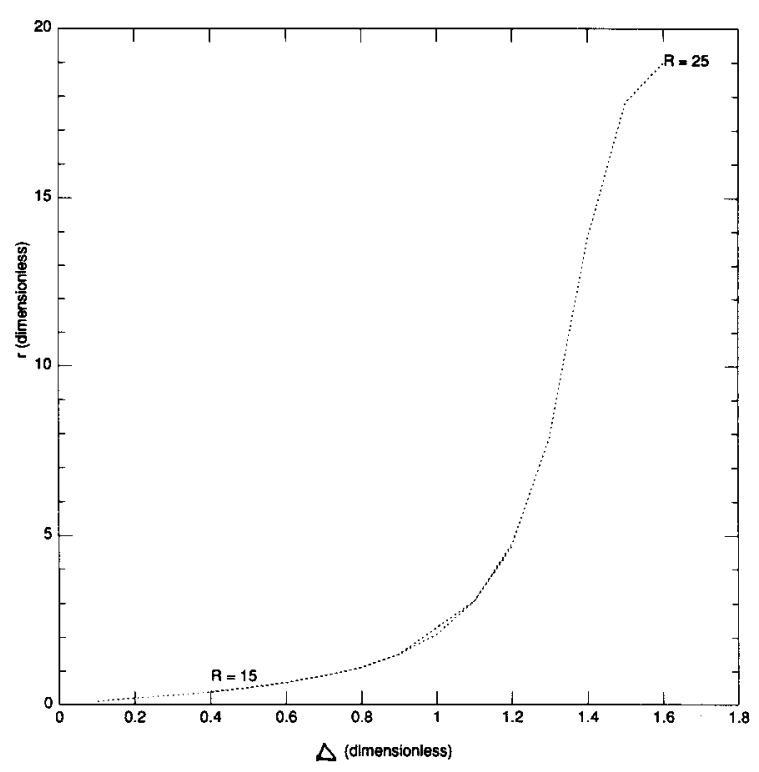

Fig. 5. A plot of the selected core radius, $r_{c}$, vs $\Delta$ for two different domain sizes, $R=15,25$. The results are identical except for high $\Delta$, where boundary effects become important.

\subsection{The small diffusion core}

Let us now consider the case $1 \gg \delta \gg \epsilon$, where it will turn out that it is still possible to formulate the core problem as an interfacial equation. Here, the diffusionless equation solved above is 
inappropriate at the core, since the $\delta \nabla^{2} v$ term becomes relevant at a length scale large compared to $\epsilon$. For this case, analyzed by Bernoff in [16], the procedure is to first use the Fife scaling, yielding the system (11), and then make the additional rescaling $\hat{r}=\tilde{r} \delta^{1 / 3}$ and $\hat{v}=\tilde{v} \delta^{-1 / 3}$. After expanding $u$ and $v$ in a power series in $\delta^{1 / 3}$, the system (11) becomes, at zeroth order in $\delta$,

$\omega^{*} \frac{\partial v_{0}}{\partial \theta}=0$

At first order, single-valuedness of $v_{1}$ in $\theta$ yields the solvability condition

$\int \mathrm{d} \theta\left(\nabla^{2} v_{0}+g^{ \pm}\right)=0$.

In this scaling limit, the Gibbs-Thomson boundary condition (10) becomes

$c_{v} v_{0}\left(r_{\mathrm{I}}\right)-\kappa\left(r_{\mathrm{I}}\right)=0$.

In general, since the two interfaces, $\theta^{ \pm}(r)$ will come together and meet at some radius $r_{c}$, the solvability condition, (32) will reduce to two different differential equations for $r\left\langle r_{\mathrm{c}}\right.$ and $\left.r\right\rangle$ $r_{\mathrm{c}}$. For $r<r_{\mathrm{c}}$, there is only the quiescent region, so (32) becomes

$\frac{\mathrm{d}^{2} v_{0}}{\mathrm{~d} r^{2}}+\frac{1}{r} \frac{\mathrm{d} v_{0}}{\mathrm{~d} r}=-g^{-}$.

The solution that remains finite at $r=0$ is given by

$v_{0}=-\frac{1}{4} g^{-} r^{2}+v_{c} \quad\left(r \leq r_{c}\right)$,

where $v_{\mathrm{c}}$ is the value of $v_{0}$ at the origin. For $r>r_{\mathrm{c}}$, (32) becomes

$$
\begin{aligned}
& \frac{\mathrm{d}^{2} v_{0}}{\mathrm{~d} r^{2}}+\frac{1}{r} \frac{\mathrm{d} v_{0}}{\mathrm{~d} r} \\
& \quad=-g^{-}-\frac{\left(g^{+}-g^{-}\right)}{2 \pi}\left[\theta^{+}(r)-\theta^{-}(r)\right] .
\end{aligned}
$$

The interfaces, $\theta^{ \pm}(r)$, and the slow field $v_{0}(r)$ for $r \geq r_{\mathrm{c}}$, are determined by (33) and (36), supplemented by boundary conditions at $r=r_{\mathrm{c}}$ and $r=\infty$. Continuity of $v_{0}$ and $v_{0}^{\prime}$ at $r=r_{\mathrm{c}}$, implies, using (35), that

$v_{0}\left(r_{\mathrm{c}}\right)=-\frac{1}{4} g^{-} r_{\mathrm{c}}^{2}+v_{\mathrm{c}}$,

$v_{0}^{\prime}\left(r_{\mathrm{c}}\right)=-\frac{1}{2} g^{-} r_{\mathrm{c}}$.

Furthermore, by symmetry, $\theta^{+}(r)=-\theta^{-}(r) \equiv$ $\theta(r)$ if we choose $\theta\left(r_{c}\right)=0$. Also, as $r \rightarrow \infty$, the core solution must match to the outer solution, i.e., $\theta(r) \rightarrow \frac{1}{2} \Delta \theta$.

It is instructive to reformulate the diffusive core problem in terms of $\theta(r)$. Using the Green function for the operator $\partial^{2} / \partial r^{2}+(1 / r)(\partial / \partial r)$,

$G\left(r, r^{\prime}\right)= \begin{cases}\ln r, & r^{\prime}<r, \\ \ln r^{\prime}, & r^{\prime}>r,\end{cases}$

we are led to the following expression for $v_{0}$ :

$v_{0}(r)=\ln r \int_{0}^{r} \tilde{R}\left(r^{\prime}\right) r^{\prime} \mathrm{d} r^{\prime}+\int_{r}^{\infty} \ln r^{\prime} \tilde{R}\left(r^{\prime}\right) r^{\prime} \mathrm{d} r^{\prime}$,

where

$$
\begin{aligned}
\tilde{R}(r) & =\frac{1}{2 \pi} \int_{0}^{2 \pi} \mathrm{d} \theta g^{ \pm} \\
& = \begin{cases}g^{-}, & r<r_{\mathrm{c}}, \\
\frac{2\left(g^{+}-g^{-}\right)}{2 \pi} \theta(r)+g^{-}, & r>r_{\mathrm{c}} .\end{cases}
\end{aligned}
$$

Note that the boundary condition $\lim _{r \rightarrow \infty} v_{0}(r)=$ 0 implies that the selected interface will have the property that $\int_{0}^{\infty} \tilde{R}\left(r^{\prime}\right) r^{\prime} \mathrm{d} r^{\prime}=0$. Geometrically, this means that the area of the excited tip will equal the area subtended by a sector of angular width $\Delta \theta$.

For numerical purposes, there are some technical difficulties with this formulation of the interface. In particular, $\theta^{\prime}(r)$ diverges as $r \rightarrow r_{c}$. It is more convenient to reformulate the problem in terms of the interfacial arclength, $s$. In this formulation, $s$ is the independent variable and $r$ becomes a new dependent variable. We also replace the variable $\psi$ with the angular coordi- 
nate $\phi$, the angle that the normal to the curve makes with the negative $x$-axis $(\theta=\pi)$. The curvature $\kappa$ is given simply in terms of $\phi$ by

$$
\kappa(s)=\phi_{s} .
$$

Here, the $s$ subscript denotes differentiation with respect to arclength. In these coordinates, we define $\mathrm{d} v_{0} / \mathrm{d} s \equiv w$, and make the rescalings

$$
\begin{aligned}
& \hat{s}=\left(\frac{-c_{v}\left(g^{+}-g^{-}\right)}{\pi}\right)^{1 / 3} s, \\
& \hat{r}=\left(\frac{-c_{v}\left(g^{+}-g^{-}\right)}{\pi}\right)^{1 / 3} r, \\
& \hat{v}=\left(\frac{c_{v}^{2} \pi}{g^{+}-g^{-}}\right)^{1 / 3} v_{0}, \\
& \hat{w}=\left(\frac{-c_{v} \pi^{2}}{\left(g^{+}-g^{-}\right)^{2}}\right)^{1 / 3} w,
\end{aligned}
$$

(33) and (36) are then equivalent to the system (dropping the hats for clarity)

$$
\begin{aligned}
& r_{s}=\sin (\phi+\theta), \quad \theta_{s}=\frac{\cos (\phi+\theta)}{r}, \\
& \phi_{s}=-v, \quad v_{s}=w, \\
& w_{s}=\left(\frac{1}{2} \Delta \theta-\theta\right) r_{s}^{2}-\frac{w r_{s}}{r}+\frac{w r \theta_{s}}{r_{s}}\left(\theta_{s}+\phi_{s}\right) .
\end{aligned}
$$

We commence solving this system by first deriving its asymptotic behaviour. At $s=0, r=r_{\mathrm{c}}$, $\phi=\theta=w=0$ and $v=\frac{1}{8} \Delta \theta r_{\mathrm{c}}^{2}+v_{\mathrm{c}}$. The derivatives at $s=0$ are also easily calculated: $\left.r_{s}\right|_{s=0}=$ $\left.v_{s}\right|_{s=0}=0,\left.\quad \theta_{s}\right|_{s=0}=1 / r_{\mathrm{c}},\left.\quad \phi_{s}\right|_{s=0}=-v\left(r_{\mathrm{c}}\right), \quad$ and $\left.w_{s}\right|_{s=0}=\frac{1}{4} \Delta \theta\left(1+\phi_{s} r_{\mathrm{c}}\right)$. This is sufficient to determine the solution in terms of the parameters $r_{\mathrm{c}}$ and $v_{\mathrm{c}}$.

The asymptotics as $s \rightarrow \infty$ are a bit more involved. In this limit, $r \rightarrow s+\Delta r$, where $\Delta r$ is a constant, $\theta \rightarrow \frac{1}{2} \Delta \theta, \phi \rightarrow \frac{1}{2}(\pi-\Delta \theta)$, and $v, w \rightarrow 0$. We denote the variations of the dependent variables from their asymptotic values as $\delta \theta, \delta \phi$, $\delta w$ and $\delta v$. We linearize (44) in these quantities, and obtain the asymptotic system

$$
\begin{aligned}
& \delta \theta_{s} \sim-\frac{\delta \theta+\delta \phi}{s}, \quad \delta \phi_{s} \sim-\delta v, \\
& \delta v_{s} \sim \delta w, \quad \delta w_{s} \sim-\delta \theta-\frac{\delta w}{s} .
\end{aligned}
$$

The $r$ equation just allows for the constant shift $\Delta r$. This system is equivalent to the fourth order ODE

$s Q_{s s s s}+Q_{s s s}+Q=0$,

where $Q=s \delta \theta, \delta \phi=-Q_{s}, \delta v=Q_{s s}$ and $\delta w=$ $Q_{s s s}$. (46) has found asymptotic behaviours

$Q(s) \sim s^{1 / 8} \exp \left(\frac{4}{3} \alpha s^{3 / 4}\right)$,

where $\alpha=( \pm 1 \pm i) / \sqrt{2}$. The final solution, in the large $s$ limit, will be a linear combination of these four modes. Since two of these modes are unbounded in this limit, we need to ensure the absence of these bad modes by adjusting the two parameters $r_{\mathrm{c}}$ and $v_{\mathrm{c}}$ appropriately. We thus expect a discrete spectrum for these parameters. We represent the total solution at infinity in polar form, replacing the two unknown coefficients for the good modes by an amplitude, $A$, and a phase $\nu$,

$Q(s) \sim A s^{1 / 8} \exp \left(-\frac{4 s^{3 / 4}}{3 \sqrt{2}}\right) \cos \left(\frac{4 s^{3 / 4}}{3 \sqrt{2}}+\nu\right)$.

We solve the full system by shooting. After making an initial guess for the five unknown quantities, $r_{\mathrm{c}}, v_{\mathrm{c}}, \Delta r, A$ and $\nu$, and using the asymptotic behaviour, (48), as an initial condition, we integrate the system from a large value of $s, s_{\max }$, to some intermediate value of $s$, $s_{\text {match }}$. We then integrate out from $s=0$ to $s_{\text {match }}$. The differences in the dependent variables at $s=s_{\text {match }}$ are functions of the five unknown constants. At values of the constants corresponding to an actual solution, these functions will vanish. This scheme is implemented by a Newton solver, seeded with our initial guess, to determine the solution.

In fig. 6, we plot $r_{\mathrm{c}}$ as a function of $v_{\mathrm{c}}$, and in fig. 7 we plot a typical tip contour, correspond- 


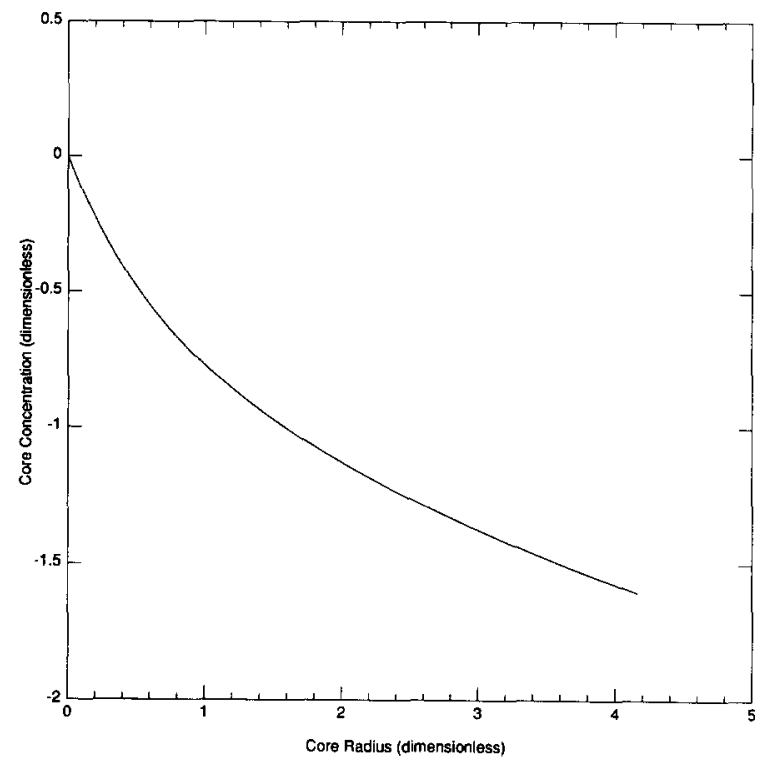

Fig. 6. A plot of the selected values of $v_{c}$ vs $r_{c}$ for the Bernoff core.

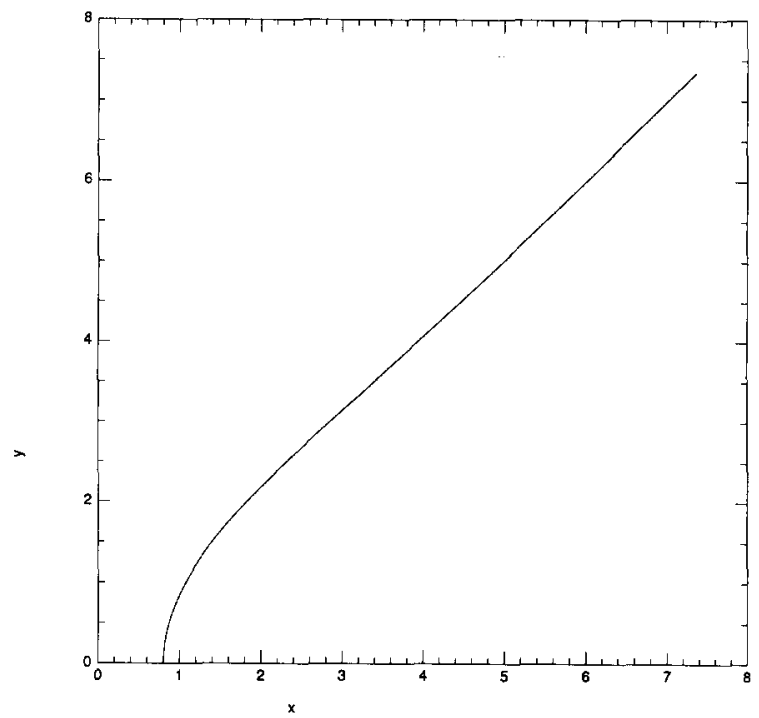

Fig. 7. A plot of a spiral tip solution for the Bernoff case $\Delta \theta=\frac{1}{4} \pi$.

ing to $\Delta \theta=\frac{1}{4} \pi$ with $v_{\mathrm{c}}=-0.673, r_{\mathrm{c}}=0.804$. In fig. 8 we plot a tip contour for $\Delta \theta=\frac{1}{10} \pi$, with $v_{0}=-1.714$ and $r_{\mathrm{c}}=4.819$. Superimposed on this plot is the angular sector $\theta=\frac{1}{10} \pi-$ this demonstrates graphically the area conservation relation $\int_{0}^{\infty} r^{\prime} \mathrm{d} r^{\prime} \tilde{R}\left(r^{\prime}\right)=0$.

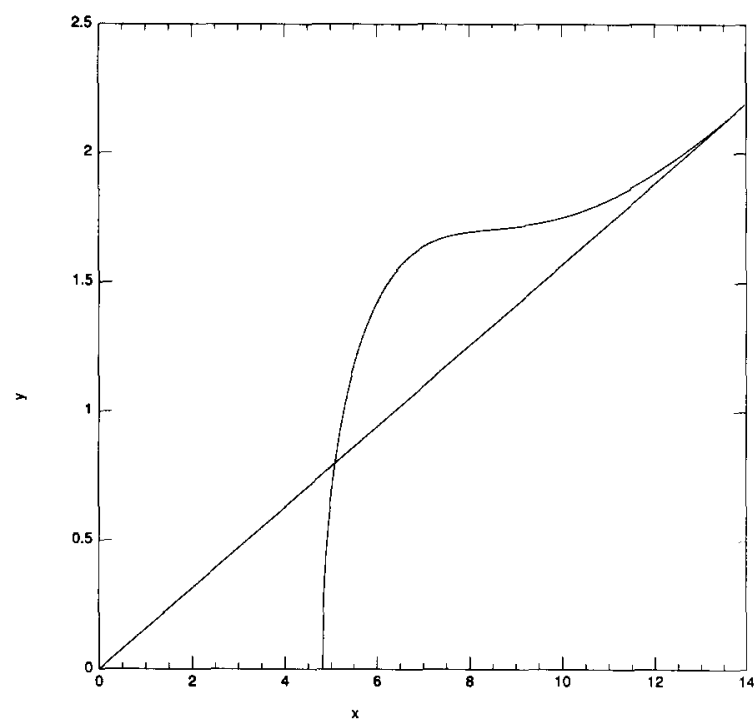

Fig. 8. A plot of a spiral tip superimposed on a sector of the same angular width. $\Delta \theta=\frac{1}{10} \pi$.

\subsection{The general case}

The two solutions described above, corresponding to $\delta=0$ and $\epsilon \ll \delta \ll 1$, are very similar - so similar in fact that the core problem begs a more general formulation. We begin such a formulation by first writing down the equations with the spatial scaling, $x=\epsilon \tilde{x}$, and Fife's temporal scaling $\partial / \partial t=-\epsilon^{-1 / 3} \omega(\partial / \partial \theta)$. The relevant parameter distinguishing the diffusionless and Bernoff cases is the ratio of $\epsilon$ to $\delta, \eta=\delta / \epsilon$. The Bernoff case corresponds to $\eta \rightarrow \infty$ and the diffusionless case corresponds to $\eta \rightarrow 0$. Using the explicit form of $g(u, v)$ for the FN model, setting $\delta=\eta \epsilon$ and dropping the tildes for clarity, we have

$$
\begin{aligned}
& -\epsilon^{2 / 3} \omega \frac{\partial u}{\partial \theta}=\nabla^{2} u+f(u, v), \\
& -\epsilon^{-1 / 3} \omega \frac{\partial v}{\partial \theta}=\eta \nabla^{2} v+u-\gamma v+\Delta .
\end{aligned}
$$

Considering the second of these equations, we have, to zeroth order,

$$
\frac{\partial v_{0}}{\partial \theta}=0
$$


which implies that $v_{0}(r, \theta)=v_{0}(r)$. At first order, we obtain the equation

$\frac{\partial v_{1}}{\partial \theta}=\eta \nabla^{2} v_{0}+u_{0}-\gamma v_{0}+\Delta$.

Single valuedness of $v_{1}$ implies that the integral of the left-hand side of this equation over $\theta$ must be 0 .

$\int_{0}^{2 \pi}\left(\eta \nabla^{2} v_{0}+u_{0}-\gamma v_{0}+\Delta\right) \mathrm{d} \theta=0$.

This leads to the equation

$$
\begin{aligned}
\nabla^{2} v_{0}-\frac{\gamma}{\eta} v_{0} & =-\frac{1}{2 \pi \eta} \int_{0}^{2 \pi}\left[u_{0}(r, \theta)+\Delta\right] \mathrm{d} \theta \\
& \equiv R(r) .
\end{aligned}
$$

The general solution to this equation is given by

$v_{0}(r)=\int_{0}^{\infty} G\left(r, r^{\prime}\right) R\left(r^{\prime}\right) r^{\prime} \mathrm{d} r^{\prime}$,

where $G\left(r, r^{\prime}\right)$ is the Green function of the operator $\partial^{2} / \partial r^{2}+(1 / r)(\partial / \partial r)-\gamma / \eta$, and is given by

$G\left(\tilde{r}, \tilde{r}^{\prime}\right)= \begin{cases}-I_{0}\left(\tilde{r}^{\prime}\right) K_{0}(\tilde{r}), & \tilde{r}>\tilde{r}^{\prime} \\ -I_{0}(\tilde{r}) K_{0}\left(\tilde{r}^{\prime}\right), & \tilde{r}^{\prime}>\tilde{r}\end{cases}$

Here, $\tilde{r}=r \sqrt{\gamma / \eta}$ and $I_{0}$ and $K_{0}$ are modified Bessel functions of the first and second kind. We now reconsider the two limiting cases previously analyzed. For the diffusionless case, $\eta \rightarrow 0$, which causes the arguments of the Bessel functions, $\sqrt{(\gamma / \eta)} r$, to become very large. Using the asymptotic behaviours, $I_{0}(x) \sim \mathrm{e}^{x} / \sqrt{2 \pi x}, K_{0}(x) \sim$ $\sqrt{(\pi / 2 x)} \mathrm{e}^{-x}(x \gg 1)$, we obtain

$$
\begin{aligned}
v_{0}(r)= & -\frac{1}{2} \sqrt{\frac{\eta}{\gamma r}} \\
& \times\left[\int_{0}^{r} \exp \left(-\sqrt{\frac{\gamma}{\eta}}\left(r-r^{\prime}\right)\right) R\left(r^{\prime}\right) \sqrt{r^{\prime}} \mathrm{d} r^{\prime}\right.
\end{aligned}
$$

$$
\left.+\int_{r}^{\infty} \exp \left(-\sqrt{\frac{\gamma}{\eta}}\left(r^{\prime}-r\right)\right) R\left(r^{\prime}\right) \sqrt{r^{\prime}} \mathrm{d} r^{\prime}\right] .
$$

To find the behaviour of the above equation in the limit of vanishing $\eta$, we note that the only significant contribution to the integrals will occur in a neighborhood of $r$ of size $\sqrt{\eta / \gamma}$, meaning that the integral form 0 to $r$ can be replaced with one from $-\infty$ to $r$. We then use the identity

$\lim _{\epsilon \rightarrow 0} \frac{\int_{x}^{\infty} \exp \left(-\left(x^{\prime}-x\right) / \epsilon\right) f\left(x^{\prime}\right) \mathrm{d} x^{\prime}}{\epsilon}=f(x)$.

As $\eta \rightarrow 0$, both integrals approach the limit $\sqrt{(\eta r / \gamma)} R(r)$. Thus we have

$v_{0}(r)=-\frac{\eta}{\gamma} R(r)$.

Inserting the explicit expression for $R(r)$, (54), we finally obtain

$v_{0}(r)=\frac{1}{2 \pi \gamma} \int_{0}^{2 \pi}\left[u_{0}(r, \theta)+\Delta\right] \mathrm{d} \theta$.

This is just (26).

For the Bernoff core, we proceed by noting that lengths should be rescaled by $\eta^{1 / 3}$ and $v$ by $\eta^{-1 / 3}$ and $\eta \rightarrow \infty$. Under this scaling, the arguments to the Green function, $(56), r \eta^{-1 / 3}$, become very small. Using the asymptotic behaviours, $I_{0} \sim 1, K_{0} \sim-\ln \left(\frac{1}{2} r\right)(r \ll 1)$, we obtain the following expression for $v_{0}(r)$ :

$$
\begin{aligned}
v_{0}(r)= & \ln \left(\frac{1}{2} r\right) \int_{0}^{r} r^{\prime} R\left(r^{\prime}\right) \mathrm{d} r^{\prime} \\
& +\int_{r}^{\infty} \ln \left(\frac{1}{2} r^{\prime}\right) R\left(r^{\prime}\right) r^{\prime} \mathrm{d} r^{\prime} \\
& +\ln \left(\sqrt{\frac{\gamma}{\eta}}\right) \int_{0}^{\infty} R\left(r^{\prime}\right) r^{\prime} \mathrm{d} r^{\prime},
\end{aligned}
$$

where $R(r)=(1 / 2 \pi) \int_{0}^{2 \pi} g\left(u_{0}, v_{0}\right) \mathrm{d} \theta$. In this sca- 
ling limit, it is easily demonstrated that $u$ and $R(r)$ approach the values of their corresponding functions from the Bernoff case. In addition, the last term in (60) vanishes, since finiteness of $v(r)$ as $r \rightarrow \infty$ implies that $\int_{0}^{\infty} R\left(r^{\prime}\right) r^{\prime} \mathrm{d} r^{\prime}=0$. Thus (60) is seen to be identical to (40). Lastly, it should be noted that a minimum principle similar to that described for the diffusionless case can be formulated, guaranteeing a solution of the inner problem in this more general case.

\section{Spiral stability}

Having determined steady spiral solutions of (1), we can now investigate their stability. In the limit we consider, the stability calculation will break up into two parts: the stability of the outer spiral solution and the stability of the core solution. Since the outer solution is identical for both the diffusive and diffusionless case, we need only calculate it once; however the stability analysis of the core region (though as we shall see, not the qualitative results) will be quite different for the two models. For the diffusionless case we must deal with the stability of the solutions of a set of coupled nonlinear field equations, whereas in the diffusive case, the problem involves an eighth order linear ODE.

\subsection{Outer solution stability}

In the outer region, we consider [34] perturbations of the steady state interface of the form $\delta \theta^{ \pm}(r) \exp (\Omega t)$. Such a perturbation will also induce changes in the $v$ field, $\delta v^{ \pm}(r, \theta) \exp (\Omega t)$. Linearizing the (Fife-scaled) equation of motion for the outer region (11), we obtain the following equation for the dynamics of $\delta v(r, \theta)$ :

$-\omega \frac{\partial \delta v^{ \pm}}{\partial \theta}+\Omega \delta v^{ \pm}=0$.

The solution to this equation is $\delta v(r, \theta)=$ $\delta A^{\ddagger}(r) \exp (\tilde{\Omega} \theta)$, where $\tilde{\Omega}=\Omega / \omega$. Now, the change in the value of the fields at the two interfaces $\delta v_{I}^{ \pm}$will have two parts, one part given by $\delta v^{ \pm}\left(\theta^{ \pm}(r)\right)$ and another induced by the shift in the interface:

$\delta v_{\mathrm{I}}^{ \pm}=\delta v^{ \pm}\left(r, \theta^{ \pm}(r)\right)+\left.\frac{\partial v}{\partial \theta}\right|_{\theta=\theta^{ \pm}(r)} \delta \theta^{ \pm}$.

This shift obviously must wind up being independent of whether it is evaluated using fields from the excited or quiescent sides of either of the two interfaces. Imposing these conditions leads to

$$
\begin{aligned}
& \delta \theta^{-}=\exp (-\tilde{\Omega} \Delta \theta) \delta \theta^{+}, \\
& \delta A^{-}=\delta A^{+}-\exp (-\tilde{\Omega} \Delta \theta) \frac{\left(g^{+}-g^{-}\right)}{\omega} \delta \theta^{+} .
\end{aligned}
$$

Our perturbed interfacial equation (10) becomes

$\delta c_{n}^{ \pm}=c_{v} \delta v_{\mathrm{I}}^{ \pm}-\delta \kappa^{ \pm}$,

where the operators $\delta c_{\mathrm{n}}[\delta \theta(r), \theta(r) ; \tilde{\Omega}, \omega]$ and $\delta \kappa[\delta \theta(r), \theta(r)]$ are the linearizations of the operators $\kappa[\theta(r)]$ and $c_{n}[\theta(r) ; \omega]$ given in (17). $\delta c_{n}$ and $\delta \kappa$ are linear in $\delta \theta$ and have opposite signs on the two interfaces. In light of (63), this implies that

$$
\begin{aligned}
& \delta \kappa^{-}=-\exp (-\tilde{\Omega} \Delta \theta) \delta \kappa^{+}, \\
& \delta c_{n}^{-}=-\exp (-\tilde{\Omega} \Delta \theta) \delta c_{n}^{+} .
\end{aligned}
$$

Using these relations in the two interfacial expressions (65), we find that the $\delta v_{\mathrm{I}}$ terms drop out, yielding the single equation

$\delta c_{n}[\delta \theta(r) ; \tilde{\Omega}]+\delta \kappa[\delta \theta(r)]=0$.

Inserting the explicit expressions for $\delta \kappa[]$ and $\delta c_{n}[; \tilde{\Omega}]$, and making the rescaling $\rho=\sqrt{\omega} r$, we obtain the following eigenvalue problem:

$$
\begin{aligned}
{\left[\delta \theta^{\prime \prime}\right.} & \left.+\delta \theta^{\prime}\left(\frac{2}{\rho}+\rho \psi-\frac{3 \psi^{\prime} \psi}{1+\psi^{2}}\right)\right]\left(\frac{1}{1+\psi^{2}}\right)-\tilde{\Omega} \delta \theta \\
& =0,
\end{aligned}
$$

with the boundary conditions that $\delta \theta$ remain 
finite as $\rho \rightarrow \infty$ and $\rho \rightarrow 0$. The second of these conditions is chosen so that the modes we are considering here do not alter the structure of the core. The stability of $\theta(r)$ is determined by the sign of the largest real part in the set of selected eigenvalues $\Omega$.

This eigenvalue problem has been solved numerically by both shooting and by explicitly diagonalizing the discretized operator. In the shooting method, we calculate the asymptotic solutions of (68) as $\rho \rightarrow \infty$, which can be shown to consist of a decaying mode, $\delta \theta \sim \exp (-\Omega \rho / B)$ and a growing mode $\delta \theta \sim \exp \left(\rho^{3} / 3 B\right)$. After picking a complex number $\Omega$, we integrate from "large" initial value of $\rho$ (typically between 4.0 and 10.0) with initial conditions that correspond to the decaying mode, towards $\rho=0$. We repeat this procedure for a grid over the complex $\Omega$ plane. Generically, both the real and imaginary parts of $\delta \theta$ diverge at $\rho=0$. We segregate the complex $\Omega$ plane by the signs of the divergence. In this way, we obtain two sets of contour lines in the $\Omega$ plane, one set where the imaginary part of $\delta \theta$ remains finite and one set for the real part. A plot of these contours is given in fig. 9. The selected values of $\Omega$ occur where these contours cross. We find that the largest real part of the selected eigenvalues is zero, which corresponds
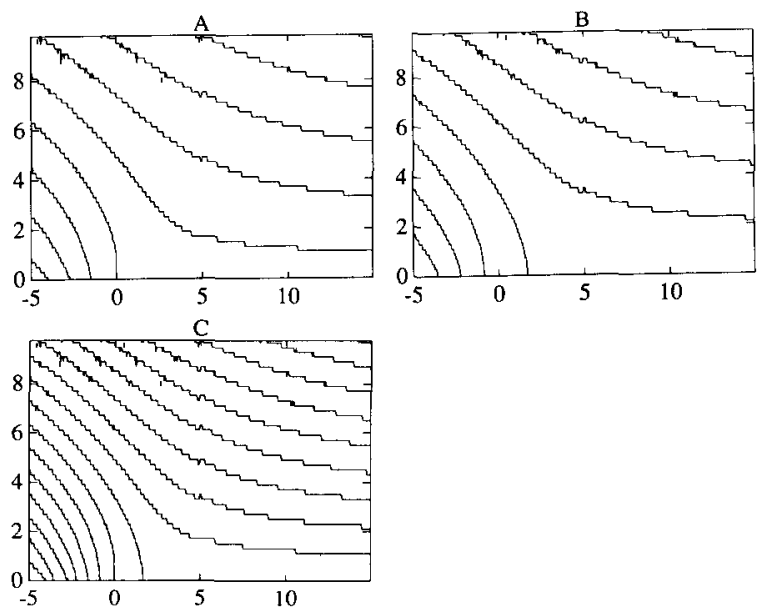

Fig. 9. Plot of the contours where $\lim _{\rho \rightarrow 0} \rho \delta \theta=0$ in the complex $\Omega$ plane. (A) Nullclines of the Real Part, (B) Nullclines of the Imaginary Part, (C) Superposition of (A) and (B). to a constant rotational shift of the entire spiral. In the explicit diagonalization approach, we approximate the stability operator with a matrix obtained by replacing the differential operators by their discrete analogues, and diagonalize. Again, the all eigenvalues are stable, except for the rotational zero mode. Spatial translation zero modes are ruled out by our insistence on finite $\delta \theta$ as $\rho \rightarrow 0$; these are more properly thought of as core modes and will play an important role later. We conclude that the outer solution to the excitable system is stable. Thus any observed instabilities must be a consequence of instabilities in the core region. Similar results have been obtained by Pelce and Sun [31].

\subsection{Core stability}

Having determined that the outer region of the steadily rotating spiral is stable, we now investigate the stability of the core region. Clearly, any stability analysis of a spiral structure that is predicated on splitting space up into two scaling regions must consider the coupling between one region and the other. In calculating the stability of the outer region, we found that the outer structure had no inherent instabilities of its own assuming that the core region was not perturbed, i.e., the boundary condition at $\rho \rightarrow 0$ was $\rho \delta \theta \rightarrow 0$. Similarly, in calculating the stability of the core region, we must account for the coupling between it and the outer region as $r \rightarrow \infty$. Such a coupling will determine the boundary condition of the stability operator in the inner region in the limit $r \rightarrow \infty$.

As we shall soon see, disturbances in the inner region, to leading order, depend on time as $\mathrm{e}^{\mathrm{i} N \omega t}$ where $N$ is an integer. Imagine solving the outer region equations (68), with this value of $\Omega$ and using the decaying mode as a boundary condition at $\infty$. As we integrate in to $\rho \sim 0, \delta \theta$ is governed by

$\frac{\partial^{2} \delta \theta}{\partial \rho^{2}} \sim-\frac{2}{\rho} \frac{\partial \delta \theta}{\partial \rho}, \quad \rho \ll 1$ 
If $N \neq 0$, the fact that $\Omega_{N}=\mathrm{i} N \omega$ is not an eigenvalue of the outer problem means that the solution diverges at the origin, with $\delta \theta_{N} \sim a_{N} / \rho+$ $b_{N}$, where $a_{N}$ and $b_{N}$ are constants. If we rescale space to the core coordinates, in either the diffusionless or the Bernoff cases, we find that that the constant term will be down by either a factor of $\epsilon^{1 / 3}$ or $\delta^{1 / 3}$, and thus negligible in the limit of either vanishing $\epsilon$ or $\delta$. So, for the case $N \neq 0$, we find that coupling between the inner and outer regions implies that the modes of the core region stability operator must asymptotically behave as $1 / \rho$. For the case $N=0, a_{0}=0$, since $\Omega_{0}=0$ is in fact an eigenvalue of the outer problem corresponding to rotation invariance of the original equations.

\subsubsection{Stability of the Bernoff curve}

We first examine the stability of the Bernoff core solution. In this case, we are investigating the stability of the solution to the set of ODEs (33), (35) and (36). We begin by considering the dynamics of a small perturbation of the form $v(r) \rightarrow v(r)+\delta v(r, \theta) \mathrm{e}^{\Omega t}, \theta^{ \pm}(r) \rightarrow \theta^{ \pm}(r)+$ $\delta \theta^{ \pm}(r) \mathrm{e}^{\Omega t}$, subject to the boundary conditions that $\delta v(r), \delta \theta^{ \pm} \rightarrow 0$ with the correct asymptotics as $r \rightarrow \infty$. After using Bernoff's scalings and linearizing (11), and then expanding $\delta v(r), \delta \theta(r)$ and $\Omega$ in power series in $\delta^{1 / 3}$, we obtain at zeroth order the following equation for $\delta v_{0}(r)$ :

$-\omega \frac{\partial \delta v_{0}}{\partial \theta}+\Omega_{0} \delta v_{0}=0$.

This implies that $\delta v_{0}(r, \theta)=\hat{v}(r) \mathrm{e}^{\mathrm{i} N \theta}$ and $\Omega_{0}=$ i $N \omega$, where $N$ is an integer. At first order, orthogonality of $\nabla^{2}\left(v_{0}+\delta v_{0}\right)+g^{ \pm}$to the adjoint null-vector, $v_{0}^{*}(r)+\delta v_{0}^{*}(r) \mathrm{e}^{-\mathrm{i} N \theta}$, leads to the equation

$$
\begin{aligned}
& \frac{\partial^{2} \hat{v}}{\partial r^{2}}+\frac{1}{r} \frac{\partial \hat{v}}{\partial r}-\left(\Omega_{1}+\frac{N^{2}}{r^{2}}\right) \hat{v} \\
& \quad=\left\{\begin{array}{cc}
0, & r<r_{\mathrm{c}}, \\
-\frac{g^{+}-g^{-}}{2 \pi}\left(\delta \theta^{+} \mathrm{e}^{-\mathrm{i} N \theta^{+}(r)}\right. & \\
\left.-\delta \theta^{-} \mathrm{e}^{\mathrm{i} N \theta^{+}(r)}\right), & r>r_{\mathrm{c}} .
\end{array}\right.
\end{aligned}
$$

Linearizing the interfacial equation (33) leads to the two equations

$$
\begin{aligned}
& \frac{\mathrm{d}^{2} \delta \theta^{ \pm}}{\mathrm{d} r^{2}}+\frac{\mathrm{d} \delta \theta^{ \pm}}{\mathrm{d} r}\left(\frac{2}{r}-\frac{3 \psi \psi^{\prime}}{1+\psi^{2}}\right) \\
& \pm \frac{c_{v}}{r}\left(1+\psi^{2}\right)^{3 / 2} \hat{v}(r) \mathrm{e}^{ \pm \mathrm{i} N \theta^{+}}=0,
\end{aligned}
$$

where $\psi(r)=r\left[\mathrm{~d} \theta^{+}(r) / \mathrm{d} r\right]$.

The stability problem is greatly simplified by realizing that the system (71), (72) is Hermitian, even for $N \neq 0$. To see this, we can explicitly solve (72) for the interface shift $\delta \theta^{+}(r)$. We rewrite (72) using an integrating factor

$$
\begin{aligned}
L\left[\delta \theta^{ \pm}(r)\right] & \equiv \frac{\mathrm{d}}{\mathrm{d} r}\left(\frac{r^{2}}{\left(1+\psi^{2}\right)^{3 / 2}} \frac{\mathrm{d} \delta \theta^{ \pm}}{\mathrm{d} r}\right) \\
& =\mp c_{v} r \hat{v}(r) \mathrm{e}^{ \pm \mathrm{i} N \theta^{+}(r)} \equiv R^{ \pm}(r) .
\end{aligned}
$$

The general solution to this equation is given by

$$
\begin{aligned}
\delta \theta^{ \pm}(r)= & -\int_{r_{\mathrm{c}}}^{\infty} R^{ \pm}\left(r^{\prime}\right) \int_{\max \left(r, r^{\prime}\right)}^{\infty} \mathrm{d} \hat{r} B(\hat{r}) \\
& +\alpha^{ \pm} \int_{r}^{\infty} B(\hat{r}) \mathrm{d} \hat{r}+\beta^{ \pm},
\end{aligned}
$$

where $B(r)=\left[1+\psi^{2}(r)\right]^{3 / 2} / r^{2}$ and $\alpha^{ \pm}$and $\beta^{ \pm}$ are arbitrary constants, representing the amplitude of the homogeneous part of the solution, and are determined by requiring that $\delta \theta^{ \pm}(r)$ satisfy the correct boundary conditions at $r=r_{\mathrm{c}}$ and $r=\infty$. At $\infty$, we require that $\delta \theta^{ \pm}$vanish, which implies $\beta^{ \pm}=0$. At $r=r_{\mathrm{c}}$, the square root singularity of $\theta^{ \pm}, \theta^{ \pm}(r) \sim \pm 2 b \sqrt{r-r_{c}}$, implies that $\delta \theta^{ \pm}$have equal and opposite inverse square root singularities, induced by the shift in the tip radius, $r_{\mathrm{c}}$. This, together with the equality of $\theta^{ \pm}$ at the tip, implies the conditions that

$\delta \theta(r)^{ \pm} \sim \pm \frac{C_{1}}{\sqrt{r-r_{c}}}+C_{2}$

as $r \rightarrow r_{c}$, where $C_{1}$ and $C_{2}$ are constants. Examining (74) near $r=r_{c}$, we see that the homogenous term indeed possesses the requisite singularity. Separating out this singularity, 
$\int_{r}^{\infty} B\left(r^{\prime}\right) \mathrm{d} r^{\prime}=\frac{2 b^{3} r_{\mathrm{c}}}{\sqrt{r-r_{\mathrm{c}}}}+\int_{r}^{\infty} B_{R}\left(r^{\prime}\right) \mathrm{d} r^{\prime}$,

where the second term is finite as $r \rightarrow r_{\mathrm{c}}$, we find that the boundary condition (75) implies

$$
\begin{aligned}
\alpha^{ \pm}= & -\frac{c_{v}}{\int_{r_{\mathrm{c}}}^{\infty} B_{R}(\hat{r}) \mathrm{d} \hat{r}} \int_{r_{\mathrm{c}}}^{\infty}\left(r^{\prime} \hat{v}\left(r^{\prime}\right) \cos \left[N \theta\left(r^{\prime}\right)\right]\right. \\
& \left.\times \int_{r^{\prime}}^{\infty} B(\hat{r}) \mathrm{d} \hat{r}\right) \mathrm{d} r^{\prime} .
\end{aligned}
$$

Substituting back in to (74), we can now use that equation to rewrite (71) entirely in terms of $\hat{v}(r)$. After making the transformation $Q(r)=\sqrt{r} \hat{v}(r)$, the eigenvalue problem, (71), becomes

$$
\begin{aligned}
& \frac{\mathrm{d}^{2} Q}{\mathrm{~d} r^{2}}-\frac{N^{2}-\frac{1}{4}}{r^{2}} Q \\
& -\frac{g^{+}-g^{-}}{2 \pi} \int_{r_{\mathrm{c}}}^{\infty} Q\left(r^{\prime}\right) \sqrt{r r^{\prime}} F\left(r, r^{\prime}\right) \mathrm{d} r^{\prime} \\
& \quad=\Omega_{1} Q,
\end{aligned}
$$

where

$$
\begin{aligned}
F\left(r, r^{\prime}\right)= & 2 c_{v}\left(\cos \left[N\left[\theta\left(r^{\prime}\right)-\theta(r)\right]\right]\right. \\
& \times \int_{\max \left(r, r^{\prime}\right)}^{\infty} B(\hat{r}) \mathrm{d} \hat{r} \\
& -\cos \left[N \theta\left(r^{\prime}\right)\right] \cos [N \theta(r)] \\
& \left.\times \frac{\int_{r^{\prime}}^{\infty} B(\hat{r}) \mathrm{d} \hat{r} \int_{r}^{\infty} B(\hat{r}) \mathrm{d} \hat{r}}{\int_{r_{\mathrm{c}}}^{\infty} B_{R}(\hat{r}) \mathrm{d} \hat{r}}\right) .
\end{aligned}
$$

Since $F\left(r, r^{\prime}\right)=F\left(r^{\prime}, r\right)$, and $F$ is real, the operator is Hermitian, implying that the eigenvalues, $\Omega_{1}$, are real, even for $N \neq 0$.

\subsubsection{Numerics of the Bernoff stability problem}

Armed with the knowledge that the selected values of $\Omega_{1}$ will be real, we can now proceed to implement a numerical scheme for finding $\Omega_{1}$. As with our base solution, (44), we express each of the perturbed interface curves in terms of its arclength, with the shifts $\delta \theta^{ \pm}(s), \delta r^{ \pm}(s)$, and $\delta \phi^{ \pm}(s)$. There is a subtlely in treating the potential $v_{0}+\delta v_{0}$, since they are most naturally expressed in terms of $r$, not $s$. The potential at a given point along the interface is given to first order by

$$
\begin{aligned}
& v_{0}\left(r(s)+\delta r^{ \pm}\right)+\hat{v}(r(s)) \mathrm{e}^{\mathrm{i} N \theta^{ \pm}(s)} \\
& \quad \approx v_{0}(r(s))+\frac{\mathrm{d} v_{0}}{\mathrm{~d} r} \delta r^{ \pm}+\hat{v}\left(r^{ \pm}(s)\right) \mathrm{e}^{ \pm \mathrm{i} N \theta(s)} .
\end{aligned}
$$

Now, $v_{0}(r(s))$ is just what we denoted as $v(s)$ in our solution of the steady-state problem. Similarly, $\delta \theta(r)$, the shift at fixed $r$, is related to $\delta \theta(s)$, the shift at fixed $s$, by

$\delta \theta^{ \pm}(r)=\delta \theta^{ \pm}(s)-\frac{\mathrm{d} \theta^{ \pm}}{\mathrm{d} r} \delta r^{ \pm}$

If we denote $\hat{v}(r(s)) \equiv \delta v(s)$, and $w \equiv \mathrm{d} \delta v / \mathrm{d} s$ we obtain, upon making the rescalings in (43), the following eighth order system:

$$
\begin{aligned}
\delta r_{s}^{ \pm}= & \pm\left(\delta \theta^{ \pm}+\delta \phi^{ \pm}\right) \cos \gamma, \\
\delta \theta_{s}^{ \pm}= & -\left(\delta \theta^{ \pm}+\delta \phi^{ \pm}\right) \frac{\sin \gamma}{r} \mp \delta r^{ \pm} \frac{\cos \gamma}{r^{2}}, \\
\delta \phi_{s}^{ \pm}= & \left(\delta v \mathrm{e}^{ \pm \mathrm{i} N \theta}+\delta r^{ \pm} \frac{w}{r_{s}}\right), \\
\delta v_{s}= & \delta w, \\
\delta w_{s}= & \delta w\left(\frac{r_{s} s}{r_{s}}-\frac{r_{s}}{r}\right)+\delta v\left(\hat{\Omega}_{1}+\frac{N^{2}}{r^{2}}\right) r_{s}^{2} \\
& -\frac{r_{s}^{2}}{2}\left[\left(\delta \theta^{+}-\delta r^{+} \frac{\theta_{s}}{r_{s}}\right) \mathrm{e}^{-i N \theta}\right. \\
& \left.-\left(\delta \theta^{-}+\delta r^{+} \frac{\theta_{s}}{r_{s}}\right) \mathrm{e}^{i N \theta}\right],
\end{aligned}
$$

where $\quad \gamma \equiv \theta+\phi \quad$ and $\quad \hat{\Omega}_{1} \equiv\left[\pi /-c_{v}\left(g^{+}-\right.\right.$ $\left.\left.g^{-}\right)\right]^{2 / 3} \Omega_{1}$.

As $s \rightarrow 0, r \sim r_{\mathrm{c}}+\left(1-v_{\mathrm{c}} r_{\mathrm{c}}\right)\left(s^{2} / 2 r_{\mathrm{c}}\right) \equiv r_{\mathrm{c}}+\eta s^{2} /$ $2 r_{\mathrm{c}}, \quad \theta \sim s / r_{\mathrm{c}}, \quad \phi \sim-v\left(r_{\mathrm{c}}\right) s, \quad v \sim v_{\mathrm{c}}+\frac{1}{8} \Delta \theta\left(r_{\mathrm{c}}^{2}+\right.$ $\left.\eta s^{2}\right), w \sim \frac{1}{4} \Delta \theta \eta s$ and $\gamma \sim \eta s / r_{\mathrm{c}}$. In this limit, (83) takes the asymptotic form 
$\delta r_{s}^{ \pm} \sim \pm\left(\delta \theta^{ \pm}+\delta \phi^{ \pm}\right)$

$\delta \theta_{s}^{ \pm} \sim \mp\left(\frac{\delta r^{ \pm}}{r_{\mathrm{c}}^{2}}\right)$,

$\delta \phi_{s}^{ \pm} \sim \mp\left(\delta v+\delta r^{ \pm} \frac{1}{4} r_{\mathrm{c}} \Delta \theta\right)$,

$\delta v_{s} \sim \delta w$,

$\delta w_{s} \sim \frac{\delta w}{s}$.

The boundary conditions at $s=0$ are that $\delta r^{+}=$ $\delta r^{-}, \delta \theta^{+}=\delta \theta^{-}, \delta \phi^{+}=\delta \phi^{-}$. In addition, the condition that $\hat{v}(r)$ and its derivative are continuous at $r=r_{\mathrm{c}}$ gives a condition relating $\delta v$ and $\delta w$ at $s=0$. For $r<r_{\mathrm{c}}$, (71) has the regular solution $\hat{v}=\hat{v}_{0} I_{0}\left(\sqrt{\Omega_{1}} r\right)$. This implies that, as $s \rightarrow$ $0, \quad \delta w(s) \approx-\left(\eta s / r_{\mathrm{c}}\right)\left[\sqrt{\Omega_{1}} I_{1}\left(\sqrt{\Omega_{1}} r_{\mathrm{c}}\right) / I_{0}\left(\sqrt{\Omega_{1}} r_{\mathrm{c}}\right)\right]$ $\delta v(0)$. We exploit the freedom in choosing the origin of $s$ by choosing $\delta \phi^{ \pm}(0)=0$. These five conditions leave three degrees of freedom in the solution, which we can identify as $\delta r^{ \pm}(0), \delta \theta^{ \pm}(0)$ and $\delta v(0)$. As this is a linear problem, we can equally well think of the solution as a linear combination of three modes, with amplitudes $\mu_{1}$, $\mu_{2}$ and $\mu_{3}$.

As $s \rightarrow \infty$, (83) has the asymptotic form

$\delta r_{s}^{ \pm} \sim 0$,

$\delta \theta_{s}^{ \pm} \sim-\frac{\delta \theta^{ \pm}+\delta \phi^{ \pm}}{s}$,

$\delta \phi_{s}^{ \pm} \sim \mp \delta v \mathrm{e}^{ \pm \mathrm{i} N \Delta \theta / 2}$,

$\delta v_{s} \sim \delta w$,

$\delta w_{s} \sim \Omega_{1} \delta v-\frac{1}{2}\left(\delta \theta^{+} \mathrm{e}^{-\mathrm{i} N \Delta \theta / 2}-\delta \theta^{-} \mathrm{e}^{+\mathrm{i} N \Delta \theta / 2}\right)$.

The equation for $\delta r^{ \pm}$decouples, and corresponds to independent constant shifts in $\delta r^{ \pm}$. The rest of the system simplifies if we introduce the variables $y^{ \pm} \equiv \delta \theta^{+} \mathrm{e}^{-\mathrm{i} N \Delta \theta / 2} \pm \delta \theta^{-} \mathrm{e}^{\mathrm{i} N \Delta \theta / 2}$ and $z^{ \pm} \equiv$ $\delta \phi^{+} \mathrm{e}^{-\mathrm{i} N \Delta \theta / 2} \pm \delta \phi^{-} \mathrm{e}^{\mathrm{i} N \Delta \theta / 2}$. Reexpressed in these variables, the rest of (85) becomes

$y_{s}^{ \pm} \sim-\frac{y^{ \pm}+z^{ \pm}}{s}$,

$z_{s}^{+} \sim 0$,

$z_{s}^{-} \sim-2 \delta v$, $\delta v_{s} \sim \delta w$

$\delta w_{s} \sim \Omega_{1} \delta v-\frac{1}{2} y^{-}$.

We see immediately that $y^{+}, z^{+}$decouple from the rest of the modes. The condition that these vanish as $s \rightarrow \infty$ eliminates one of the modes, leaving the mode $y^{+} \sim s^{-1}, z^{+}=0$. The other equations give rise to four modes, with asymptotic behaviours $\mathrm{e}^{ \pm \sqrt{\Omega_{1} s}}$ and $\mathrm{e}^{ \pm \sqrt{s / \Omega_{1}}}$. For positive real $\Omega_{1}$, two of these are growing modes and therefore unacceptable, leaving us with an additional two modes. There is thus a grand total of five modes, counting the constant (to leading order in $s$ ) shifts in $\delta r^{ \pm}$, which we can parametrize by the amplitudes $\nu_{i}, i=1, \ldots, 5$.

We have seen that as $s \rightarrow \infty$, (83) has five independent modes, which we can label $\nu_{i}(s)$, with amplitudes $\nu_{i}$. Note that each of the $\nu_{i}(s)$ is an eight-dimensional vector, corresponding to the eight functions in (83). Likewise, as $s \rightarrow 0$, there are three independent modes, $\mu_{j}(s)$ with amplitudes, $\mu_{j}$. An eigenmode of the system exists [30] if, for some values of the $\nu_{i}$ determining the solution at large $s$, after integrating in from infinity to some arbitrary intermediate value of $s, s_{\text {match }}$, the solution is continuous with the result of integrating the system out from 0 to $s_{\text {match }}$ for some choice of the $\mu_{j}$. Since this is a linear problem, we can express the results of the integration in from infinity as a linear combination of the vectors $\boldsymbol{\nu}_{i}\left(s_{\text {match }}\right)$. Similarly, the result of integrating in from 0 is a linear combination of the $\boldsymbol{\mu}_{j}\left(s_{\text {match }}\right)$. Continuity requires that eight equations be satisfied

$\sum_{i} \nu_{i} \nu_{i}\left(s_{\text {match }}\right)=\sum_{j} \mu_{j} \mu_{j}\left(s_{\text {match }}\right)$.

As this system is homogenous, it must be degenerate for a non-trivial solution to exist. This degeneracy requirement

$$
\begin{aligned}
\Phi\left(\Omega_{1}\right) & \equiv \operatorname{det}\left[\mu_{1}, \mu_{2}, \mu_{3}, \nu_{1}, \nu_{2}, \nu_{3}, \nu_{4}, \nu_{5}\right]\left(s_{\text {match }}\right) \\
& =0
\end{aligned}
$$

gives a selection criterion for the eigenvalue $\Omega_{1}$.

Our major result is presented in fig. 10 , where 


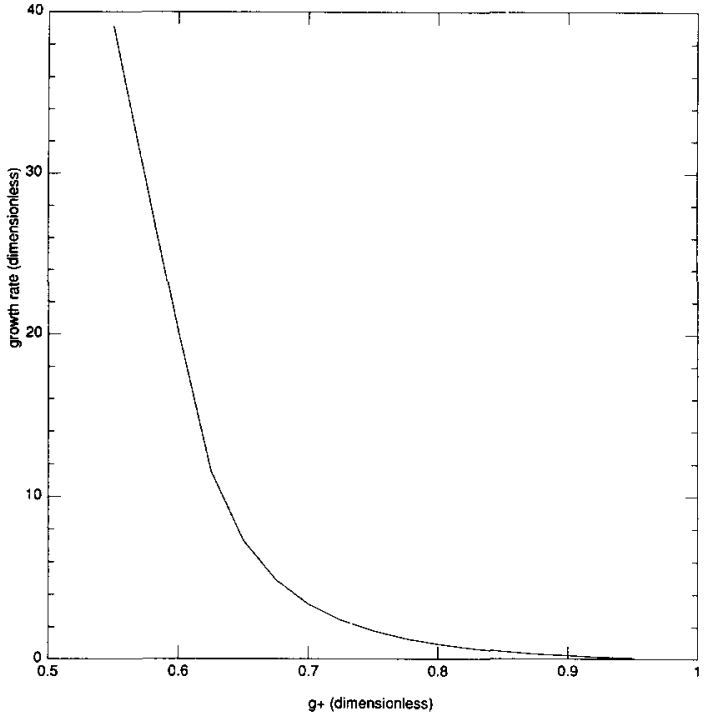

Fig. 10. Plot of the selected eigenvalues for the Bernoff core stability problem vs $g^{+}$.

we have plotted the positive values of $\Omega_{1}$ for $N=0$ at different values of $g^{+}$; for simplicity, we have scaled $-c_{v}=g^{+}-g^{-}=1$, leaving $g^{+}$as the only parameter. There is one mode which is always unstable and whose growth rate diverges as we approach $g^{+}=\frac{1}{2}$, which is of course the symmetric case $\theta_{+}=-\theta_{-}=\pi, r_{c}=0$. There are no instabilities for other $N$; in fact, if we artificially extend the integer $N$ in the stability operator to be a real number, the real part of the growth rate of the $N=0$ unstable mode decreases uniformly as $N$ increases, crossing zero at precisely $N=1$. A plot of the growth rate vs $N$ is given in fig. 11. The existence of a mode at precisely $\Omega=i \omega$ is due to the translation invariance of the underlying dynamics. This provides a highly non-trivial check on the details of our calculation. The upshot of all this is that the core exhibits one unstable mode!

\subsubsection{Stability of the diffusionless core}

The diffusionless core is described by (21), (23), (24). To examine the stability of this solution, we consider the effects of a small perturbation of the form $u \rightarrow u+\delta u(r, \theta) \mathrm{e}^{\Omega t}$,

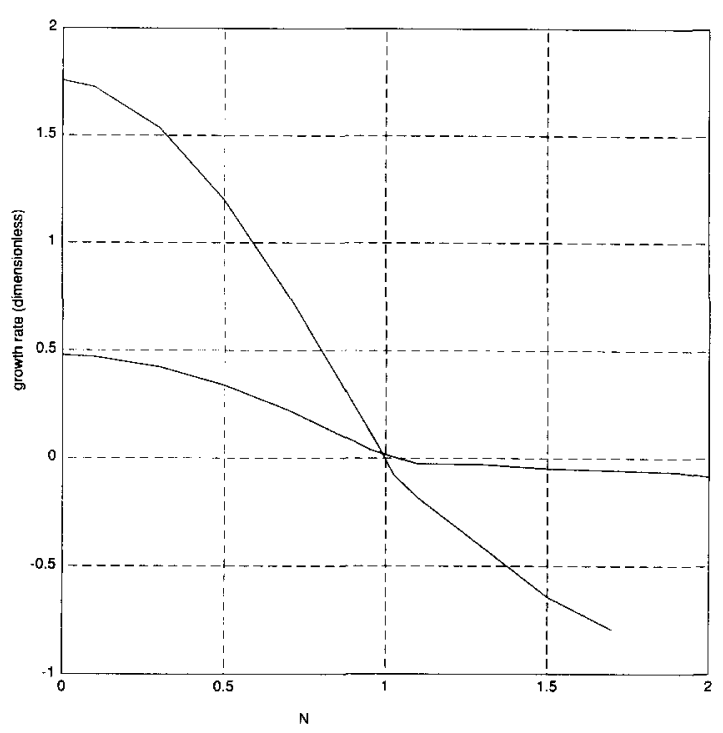

Fig. 11. Plot of the selected eigenvalues at $g^{+}=0.75,0.85$ as function of $N$. Note the zero crossing at $N=1$.

$v \rightarrow v+\delta v(r, \theta) \mathrm{e}^{\Omega t}$. We expand the perturbations in a power series in $\epsilon^{1 / 3}$,

$\delta u=\delta u_{0}+\epsilon^{1 / 3} \delta u_{1}+\mathcal{O}\left(\epsilon^{2 / 3}\right)$,

$\delta v=\delta v_{0}+\epsilon^{1 / 3} \delta v_{1}+\mathscr{O}\left(\epsilon^{2 / 3}\right)$,

$\Omega=\Omega_{0}+\epsilon^{1 / 3} \Omega_{1}+\mathcal{O}\left(\epsilon^{2 / 3}\right)$.

Using (1), and the spatial and temporal scalings appropriate to the core region, this yields at zeroth order the linear system:

$$
\begin{aligned}
& \nabla^{2} \delta u_{0}+\left.\frac{\partial f}{\partial u}\right|_{u_{0}, v_{0}} \delta u_{0}+\left.\frac{\partial f}{\partial v}\right|_{u_{0}, v_{0}} \delta v_{0}=0, \\
& -\omega \frac{\partial \delta v_{0}}{\partial \theta}+\Omega_{0} \delta v_{0}=0 .
\end{aligned}
$$

Eq. (92) implies

$$
\dot{\delta v_{0}}(r, \theta)=\delta v_{0}^{N}(r) \exp \left(\frac{\Omega_{0}}{\omega} \theta\right) .
$$

Single valuedness of $v_{0}$ implies that $\Omega_{0}=$ i $N \omega \equiv \Omega_{0}^{N}$, where $N$ is an integer. Thus, finding the spectrum of this system decomposes into a separate eigenvalue problem for each value of $N$. The eigenmodes will either grow or decay at a 
(slow) rate proportional to $\epsilon^{1 / 3}$, depending upon the sign of $\Omega_{1}$ for the given mode.

To explicitly obtain the relevant problem we first determine $\delta v_{0}^{N}(r)$ by expanding (1) to first order,

$$
\begin{aligned}
- & \omega \frac{\partial \delta v_{1}^{N}}{\partial \theta}+\Omega_{0}^{N} \delta v_{1}^{N} \\
= & \left.\frac{\partial g}{\partial u}\right|_{u_{0}, v_{0}} \delta u_{0}^{N}+\left(\left.\frac{\partial g}{\partial v}\right|_{u_{0}, v_{0}}-\Omega_{1}^{N}\right) \delta v_{0} .
\end{aligned}
$$

We have appended the superscript $N$ to both $\delta u_{0}$ and $\Omega_{1}$ to denote that we are considering some fixed $N$. We use (92) to substitute for $\delta v_{0}$ and multiply by $\exp (-\mathrm{i} N \theta)$. Integrating over $\theta$ leads to the solvability condition

$$
\begin{gathered}
\int_{0}^{2 \pi} \frac{\mathrm{d} \theta}{2 \pi}\left(\left.\mathrm{e}^{-\mathrm{i} N \theta} \frac{\partial g}{\partial u}\right|_{u_{0}, v_{0}} \delta u_{0}^{N}\right) \\
=\left(\Omega_{1}^{N}-\left.\frac{\partial g}{\partial v}\right|_{u_{0}, v_{0}}\right) \delta v_{0}^{N}(r) .
\end{gathered}
$$

Specializing now to the FN model, we obtain the following expression for $\delta v_{0}^{N}(r)$ :

$\delta v_{0}^{N}(r)=\left(\frac{1}{\Omega_{1}^{N}+\gamma}\right) \int_{0}^{2 \pi} \mathrm{e}^{-\mathrm{i} N \theta^{\prime}} \delta u_{0}^{N}\left(r, \theta^{\prime}\right) \frac{\mathrm{d} \theta^{\prime}}{2 \pi}$.

Using this expression for $\delta v_{0}^{N}(r)$ in (90), we obtain the following eigenvalue problem for the perturbation $\delta u_{0}^{N}(r, \theta)$ :

$$
\begin{aligned}
& \nabla^{2} \delta u_{0}^{N}+\left.\frac{\partial f}{\partial u}\right|_{u_{0}, v_{0}} \delta u_{0}^{N} \\
& =\left(\frac{1}{\Omega_{1}^{N}+\gamma}\right) \int_{0}^{2 \pi} \mathrm{e}^{-\mathrm{i} N \theta^{\prime}} \delta u_{0}^{N}\left(r, \theta^{\prime}\right) \frac{\mathrm{d} \theta^{\prime}}{2 \pi} .
\end{aligned}
$$

We would expect that any spiral solution, and hence any core solution would be translationally symmetric, since there is nothing that distinguishes the medium in the vicinity of the core from the medium in the rest of space. This implies that the perturbations $\delta u_{0}, \delta v_{0}$ corresponding to translations should exhibit vanishing growth rates. Since the core solution is rotating with an angular velocity $\omega$, such a translational mode will when viewed in the rotating frame have $N=1$. In fact, it is easy to see that a translational mode will have the explicit form

$\delta u_{0}(r, \theta) \propto \mathrm{e}^{\mathrm{i} \theta}\left(\frac{\partial u_{0}}{\partial r}+\frac{\mathrm{i}}{r} \frac{\partial u_{0}}{\partial \theta}\right)$.

If we now insert this quantity into (96) and set $\tilde{\Omega}_{1}^{N}=0$, we can verify that the equation is satisfied identically.

One important consideration for this eigensystem is the issue of the expected asymptotic behaviour for $\delta u_{0}^{N}(r)$ as $r \rightarrow \infty$. The answer to this question is similar to that discussed in the last section for the perturbation to the Bernoff core. That is, the perturbation must match smoothly onto the outer perturbation, which consists of the motion of the thin interface by an amount that varies as $1 / r$ at small $r$ in the outer variables. This implies that our perturbation must behave as $\delta u_{N}^{0} \sim \mathrm{i}\left(\mathrm{e}^{\mathrm{i} N \theta} / r\right)\left(\partial u_{0} / \partial \theta\right)$ for large $r$. This provides a boundary condition for the perturbation $\delta u_{0}$ for all cases with $N \neq 0$. Note that the translation mode given above clearly obeys this boundary condition (for $N=1$ ) since $\partial u_{0} / \partial r$ approaches zero exponentially for large $r$. The coefficient of the above relation can be fixed so as to normalize the eigenfunctions. Again the situation is different for $N=0$; here the outer solution at $\Omega_{0}$ corresponds to a pure rotation zero mode and does not couple to the non-trivial $N=0$ modes; these modes should decay rapidly to zero. For these modes, we simply set $\delta u_{0}^{0}(r, \theta)$ to zero on the edge of the (large) domain of integration. Using these boundary conditions, the solution to (96) will determine the eigenvalues $\Omega_{1}^{N}$, for each value of $N$.

We proceed by expanding $\delta u_{0}^{N}(r, \theta)$ and $\partial f(r$, $\theta) /\left.\partial u\right|_{u_{0}, v_{0}}$ in a Fourier series in $\theta$ :

$$
\begin{aligned}
& \left.\frac{\partial f(u, v)}{\partial u}\right|_{u_{0}, v_{0}}=\sum_{m=-\infty}^{\infty} f_{m}(r) \mathrm{e}^{\mathrm{i} m \theta}, \\
& \delta u_{0}^{N}(r, \theta)=\sum_{m=-\infty}^{\infty} y_{m}^{N}(r) \mathrm{e}^{\mathrm{i} m \theta} .
\end{aligned}
$$


Eq. (97) then becomes

$\nabla_{m}^{2} y_{m}^{N}+\sum_{i} f_{m-i} y_{i}^{N}=\lambda_{N} y_{N}^{N} \delta_{m N}$

where $\lambda_{N}=1 /\left(\Omega_{1}^{N}+\gamma\right), \nabla_{m}^{2}$ is the Laplacian operator for the $m$ th angular mode and $\delta_{m N}$ is the Kronecker delta. We can write this equation in bra-ket notation as

$L\left|y^{N}\right\rangle=\lambda_{N}|N\rangle\left\langle N \mid y^{N}\right\rangle$,

where $\quad\left\langle m|L| y^{N}\right\rangle=\nabla_{m}^{2}\left\langle m \mid y^{N}\right\rangle+\Sigma_{m^{\prime}} f_{m-m^{\prime}} \times$ $\left\langle m^{\prime} \mid y^{N}\right\rangle$. Operating on the left with $L^{-1}$ and taking the inner product with $\left\langle m^{\prime}\right|$ yields

$\left\langle m^{\prime}\left|L^{-1}\right| N\right\rangle\left\langle N \mid y^{N}\right\rangle=\tilde{\lambda}_{N}\left\langle m^{\prime} \mid y^{N}\right\rangle$,

where $\tilde{\lambda}_{N}=1 / \lambda_{N}=\Omega_{1}^{N}+\gamma$. This is an infinite number of equations for the modes $\left\langle m^{\prime} \mid y^{N}\right\rangle$ and the eigenvalue $\tilde{\lambda}_{N}$. We determine them by considering the $N$ th equation

$$
\left\langle N\left|L^{-1}\right| N\right\rangle\left\langle N \mid y^{N}\right\rangle=\tilde{\lambda}_{N}\left\langle N \mid y^{N}\right\rangle .
$$

This is a standard eigenvalue problem for $\tilde{\lambda}_{N}$ and $\left\langle N \mid y^{N}\right\rangle$. We may then use (101) to determine the rest of the $\left\langle m \mid y^{N}\right\rangle$. The stability problem then reduces to determining the $N N$ diagonal elements of the inverse operator $L^{-1}$, and solving the eigenvalue problem (102).

Eq. (100) is very much like a Schrödinger equation, except that the right side is a projection onto the $N$ th component of the eigenvector, rather than the full eigenvector itself. Nevertheless, we shall make an analogy to quantum mechanics to help understand the nature of the problem. In this analogy, the Hamiltonian corresponds to the operator $-L$, with energies given by $E=-1 /\left(\Omega_{1}+\gamma\right)$ which corresponds to a particle moving in the potential described by $-\partial f(u, v[u]) / \partial u$. Geometrically, this potential takes the form of a "ditch" dug out along the interface, as shown in fig. 12. Unstable modes should correspond to the bound states of this potential. Moreover, as in problems in cylindrical geometry in quantum mechanics, we should find the states with the lowest energy, i.e., the most unstable modes, have the lowest value of $N$. As we have already seen in the Bernoff case, this is precisely what we observe.

To solve this eigenvalue problem numerically, we map the problem (102) to a matrix inversion problem. We begin by truncating the Fourier expansions (98) at a maximum mode number $M$, and discretize the interval $\left[0, R_{m}\right]$ into $N_{r}$ pieces of size $\Delta r=R_{m} / N_{r}$. The asymmetric terms in the Laplacian are then removed by the transformation $\left\langle m \mid y^{N}\right\rangle=y_{m}^{N} \equiv Q_{m}^{N} / \sqrt{r}$. This leads to the following linear system for the eigenvalues:

$$
\left\langle N\left|A^{-1}\right| N\right\rangle Q_{N}^{N}=\tilde{\lambda}_{N} Q_{N}^{N} .
$$

The matrix $A$ is just the similarity transformed $L$; it is a sparse, symmetric, block structured matrix of size $(2 M+1)^{2} N_{r}^{2}$. There are $(2 M+$ $1) \times(2 M+1)$ blocks, $A^{m n}$, each of size $N_{r} \times N_{r}$. The off-diagonal blocks are diagonal, with $i$ th element $A_{i i}^{m n}=f_{m-n}\left(r_{i}\right)$ where $r_{i}=i \Delta r$. The diagonal blocks are tridiagonal, with elements $A_{i i \pm 1}^{m m}=1 /(\Delta r)^{2} \quad$ and $\quad A_{i i}^{m m}=f_{0}\left(r_{i}\right)-2 /(\Delta r)^{2}+$ $\left(\frac{1}{4}-m^{2}\right) / r_{i}^{2}$. The exceptions to this are the corner elements, $A_{11}^{m m}$ and $A_{N_{r} N_{r}}^{m m}$. Here, the values are chosen such that the ratio, $Q_{m}^{N}\left(r_{I+1}\right)$ / $Q_{m}^{N}\left(r_{l}\right)$, satisfies the correct boundary condition. In the case of $I=1$, this is given by $Q_{m}^{N}\left(r_{2}\right) /$ $Q_{m}^{N}\left(r_{1}\right)=2^{m+1 / 2}$ and $A_{11}^{m m}=2^{m+1 / 2} /(\Delta r)^{2}$, which corresponds to the small $r$ behaviour $Q_{m}^{N}(r) \sim$ $r^{m+1 / 2}$. The case $I=N_{r}$ is a little more complicated. If $N \neq 0$, the large $r$ behaviour is

$Q_{m}^{N}(r) \sim \sqrt{r}\left(\frac{\partial u_{m-N}}{\partial r}-\frac{(m-N) u_{m-N}}{r}\right)$,

where $u_{m}(r)$ is the $m$ th Fourier mode of the base solution $u_{0}(r, \theta)$. In this case, we set $A_{N_{r} N_{r}}^{m m}=$ $Q_{m}^{N}\left(r N_{r-1}\right) /(\Delta r)^{2} Q_{m}^{N}\left(r N_{r}\right)$ and the off diagonal elements $A_{N_{r} N_{r}}^{m n}$ were all set to 0 . If we are interested in the $N=0$ modes, then we simply set $Q_{m}^{0}\left(r_{N_{r}+1}\right)=0$, in which case the corner matrix elements are unchanged.

In order to determine the stability of the $N$ th mode, the matrix $A$ was first inverted using the sparse equation solver ma28, from the Harwell 


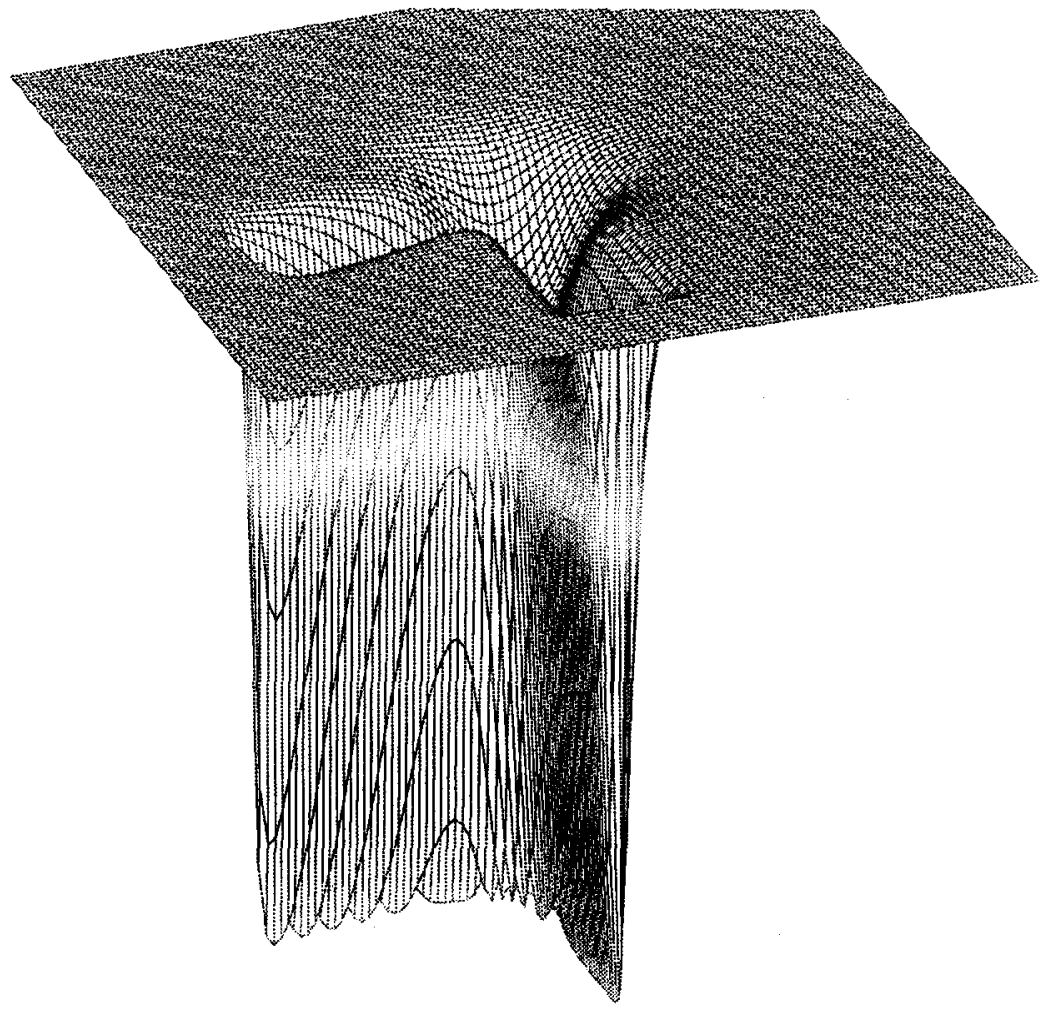

Fig. 12. Plot of the potential "ditch" whose bound states will correspond to the unstable modes of the diffusionless core.

software library [32]. For input data, core solutions were generated on grids of radius, $R_{\max }=$ 8,15 and 20 spatial units using the simple relaxational solver described in section 3.1. The stability program calculated the Fourier modes of the base solution, $u_{m}(r)$, and $f_{m}(r)=(\partial f /$ $\partial u)(r)$, and then calculated the matrix elements $A_{i j}^{m n}$. Then ma28 was called to generate $A^{-1}$. The EISPACK [33] library was used to calculate the eigenvalues $\tilde{\lambda}_{N}=\Omega_{1}^{N}+\gamma$ and eigenmodes $Q_{N}^{N}$ of the $N$ th diagonal block of $A^{-1}$. Finally, the remaining components of the $Q^{N}$ vector are found by substituting back into eq. (101) for $m^{\prime} \neq N$.

The stability program itself operates only on a sub-domain of the input base solution of radius $R$, which would generally range from $R=\frac{1}{2} R_{\max }$ to $R=\frac{4}{5} R_{\max }$. Using too much of the domain of the base solution was avoided to prevent boundary effects. In addition to operating over differ- ent domains, the program also used different discretizations in $r, \Delta r=R / N_{r}$ and $M$, the truncation number of the Fourier modes. $N_{r}$ was typically 40,60 or 80 . We used $M=30$ almost exclusively, since we found that increasing $M$ above this number had little effect on the final answer.

Ideally, the calculated growth rates could be extrapolated to infinite domain size with infinite resolution to given precise answers. Unfortunately, we found this to be well beyond the limit of our computational capability. Instead we will satisfied with determining the generic behaviour of our stability operator, specifically with regard to the $N$ dependence of the eigenvalues. We then argue, using the analogy with the Bernoff calculation, that the diffusionless core also exhibits one $N=0$ unstable mode.

Typically for $N=0$ and $N=1$, there is one eigenmode with $\tilde{\lambda}_{N}>0$. For $N=1$, we would 
Table 1

The following sampling of data was taken over a domain of size $R=15.0$, with $\gamma=0.5$. In almost all cases, $\tilde{\lambda}_{0}>\tilde{\lambda}_{1}$. For almost all observed $N>1, \tilde{\lambda}_{N}<\tilde{\lambda}_{1}$.

\begin{tabular}{lrlll}
\hline$\Delta$ & $R_{\max }$ & $N_{r}$ & $\tilde{\lambda}_{1}$ & $\tilde{\lambda}_{0}$ \\
\hline 0.6 & 10.0 & 60 & 3.569746 & 18.033898 \\
0.7 & 6.0 & 80 & 1.328298 & 11.295523 \\
0.7 & 10.0 & 60 & 1.701528 & 6.582514 \\
0.8 & 6.0 & 40 & 1.022715 & 4.665024 \\
0.8 & 8.0 & 60 & 1.240349 & 4.068046 \\
0.9 & 5.0 & 60 & 0.845676 & 4.074401 \\
0.9 & 10.0 & 80 & 1.201626 & 2.538839 \\
1.0 & 10.0 & 80 & 1.089761 & 1.804864 \\
1.1 & 6.0 & 80 & 0.747468 & 2.714109 \\
1.2 & 8.0 & 60 & 1.977321 & 1.968943 \\
1.2 & 10.0 & 60 & 1.021205 & 1.356067 \\
\hline
\end{tabular}

expect this to correspond to the translational mode and to have eigenvalue $\tilde{\lambda}=\gamma$. As can be seen from table 1 , this is clearly not the case at any degree of accuracy. One could try to postulate convergence in $N_{r}$ and the radius cutoff, but our data is not really accurate enough for that either. As already mentioned, we will instead focus on qualitative features. Most importantly, $N=0$ has a selected eigenvalue that is (almost) always greater than the $N=1$ eigenvalue. The only anomalous case occurs for very large $\Delta$, (see (3)) at too small a value of $R$; at large $\Delta$ the core "melts back" to the large $r$ outer solution by a large amount and $R=8$ is not sufficient for even qualitative behaviour - this can be seen by examining the effects of increasing $R$ which restore the standard behaviour. Also, taking very large $\Delta$ brings us close to the limit for which we expect the two eigenvalues to be extremely close in value. Hence, if we assume that for a given set of numerical parameters that the value of $\tilde{\lambda}_{1}$ corresponded to $\Omega_{1}=0$, then we could infer that the $N=0$ modes are unstable. To check this supposition, we have also calculated the eigenvalues for higher $N$ values. In all cases where the numbers can be trusted, $\tilde{\lambda}_{N}<$ $\tilde{\lambda}_{1}$.

In order to provide additional evidence that the $N=1$ modes really can be associated with a translational mode, we note that the first Fourier component of the translational mode (98) will be given by

$y_{1}^{1}(r) \propto \int \frac{\partial u^{0}}{\partial r} \delta \theta$

Therefore, we should find that mode $y^{1}(r)$ produced by the stability program with $N=1$ and translational boundary conditions should just be the $r$ derivative of $\int u^{0}(r), \bar{u}_{r}$. In fig. 13 , we see superimposed the first Fourier component of the translational mode and $y_{1}^{1}(r)$. They are almost identical. A comparison of some of the other Fourier components is given in fig. 14. Note that although the qualitative match is good, the quantitative one is not. This is not surprising if one considers how these modes are generated. In addition to errors in $y_{1}(r)$ (i.e., because of errors in the diagonal block $\left.\left(A^{-1}\right)^{11}\right)$ affecting the determination of other solution modes, we would also expect errors in the (1n) off-diagonal block of $A^{-1}$ to influence the $n$th component of the translational mode; this follows directly from eq. (101). One can verify that this latter effect is

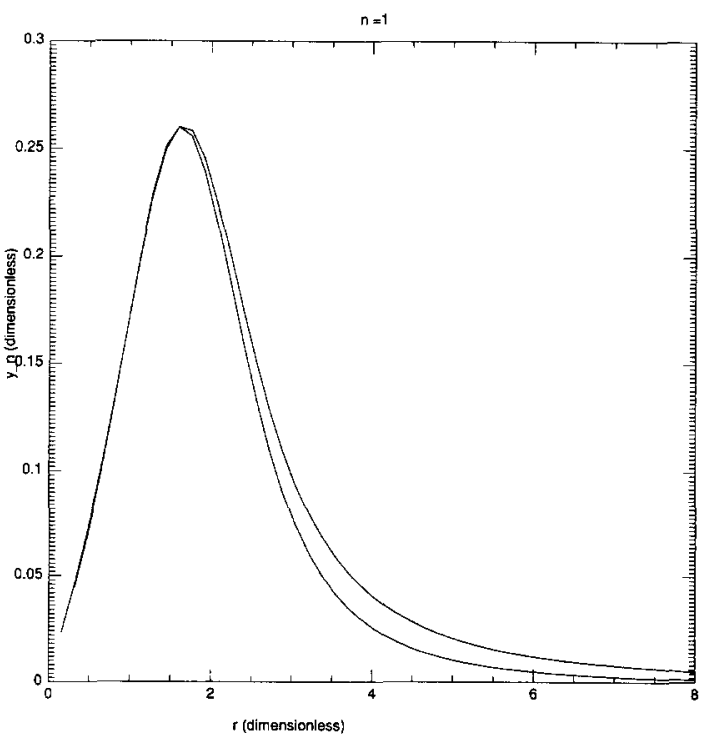

Fig. 13. Superposition of first calculated Fourier mode vs the true translational mode. The upper curve is the calculated mode, the lower the true mode. 

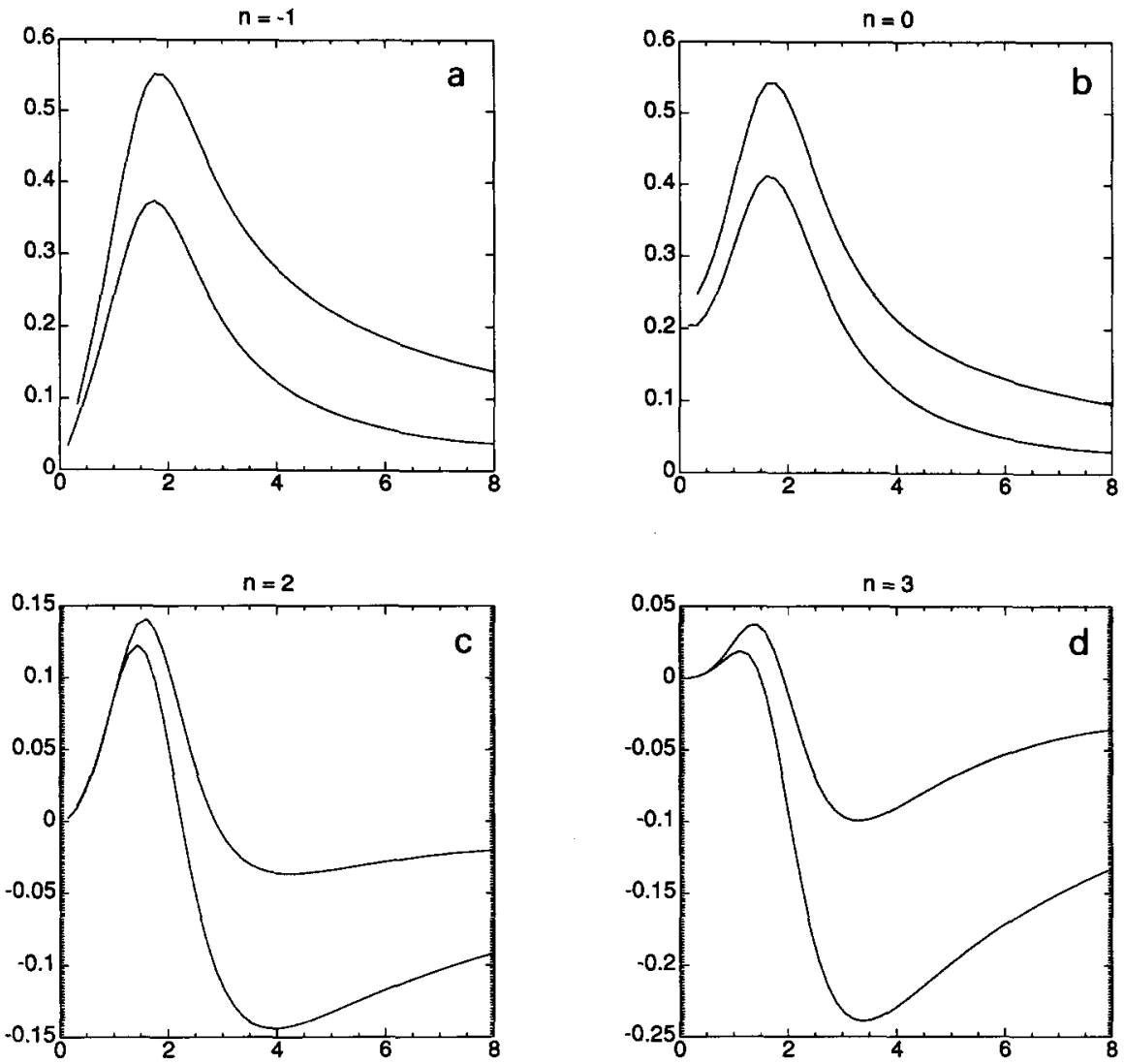

Fig. 14. Comparison of several different components of the calculated Fourier components of the calculated translational mode and the true translational mode. The axes are the same as in fig. 5 . For $n=2$ and 3 , the true mode is the lower one. For $n=0$ and -1 , the true mode is the upper one.

dominant by comparing the Fourier components of the computed translational mode to those generated by operating on the exact translational mode with $\left(A^{-1}\right)^{1 n}$.

One crucial question concerns why our computation suffers from quantitative inaccuracy. We surmise that the inaccuracy of the selected eigenvalue is due to two factors. The first is discretization error, which tends to make the selected eigenvalue too large. We see in table 1 that as $N_{r}$ is increased, the selected eigenvalue decreases. Note that we cannot decrease this error indefinitely: for values of $N_{r}$ too large, numerical noise in the base solution begins to overwhelm the selected modes. In practice, it was found that $N_{r}=80$ was the maximum value that gave reasonable behaviour. The second factor is error due to the truncation of the Fourier expansion at some finite number of modes. This error is intimately related to the size of the domains, since at large $r$ the solution becomes a step function in $\theta$, which necessitates the inclusion of many higher modes, as well as infinite spatial resolution in the base solution. Thus for increasing $R_{\max }$, we expect a degradation in the accuracy of the solution. So, we basically cannot get a domain large enough to give accurate answers since the number of points and number of Fourier modes that would be needed to discretize the operator in that domain is too large. Nevertheless, we do feel that our answers are qualitatively valid and that trends such as the 
decrease with $N$ and the existence of a translational mode should be believed.

By itself, a numerical calculation of such an imprecise nature as this would not be a compelling piece of evidence supporting the claim that an $N=0$ mode is the only core-localized instability of a spiral structure. However, one must consider these results in tandem with the numerical results of the small diffusion core. Although the numerical techniques used to generate them were completely different, the structure of the resulting eigenvalue distribution winds up being rather similar. It is therefore reasonable to assume that the highest $N=1$ mode would be precisely at $\Omega_{1}=0$ if we could get a quantitative extrapolation, and that the modes uniformly grow with $N$. Therefore, the diffusionless core exhibits an real instability which is similar to the one observed in the Bernoff limit.

\section{Discussion}

To summarize, we have discussed solutions to two component reaction-diffusion systems governing excitable media. We have worked in the limit of vanishing ratio of the reaction rates for the two species and in the cases of either zero or small diffusion in the slow field. At the crux of the both solutions is the splitting up of space into two regions: the outer region, which consists of the spiral proper, is independent of slow field diffusivity, exhibits a singularity at the spiral tip, and obeys the Fife scaling; and the inner region, whose scaling is small enough to smooth out the singularity exhibited by the outer region and which must asymptotically match onto it. A stability analysis was performed on the outer region of the spiral, assuming a stable core, with result that no instability was found. The stability of the core region was then calculated for both the small and zero diffusion cases, with result that both regions were found to have unstable modes, with purely real growth rates. Other core modes, characterized by an integer mode number, $N$, and frequency $N \omega$, were also investigated and found to be stable.

An important implication of this work is the lack of dependence of the spiral's behaviour on the precise details of the excitable media kinetics. The core solution and its stability are qualitatively independent of the particular chemical kinetics chosen. In addition, our solutions for two different types of media, diffusionless and small-diffusion, are qualitatively almost identical.

Let us now return to the question first touched upon in the introduction, namely how do these results fit into other research on spiral formation in excitable media? Simulations [23] have shown that the meandering instability is peaked about the spiral tip. One can even develop a phenomenological approach to the nature of the bifurcating mode [34], which agrees quantitatively with this numerical work. It has further been shown that the behaviour of spirals in doublydiffusive media are virtually identical to those with zero diffusion. Finally, meandering has been observed in a variety of both models and experimental systems, lending support to the claimed universality of the instability.

However, a shortcoming of the current theory is that it has little to say about the precise nature of the transition to meandering. This is due to the fact that the theory relies on a perturbation expansion in the parameter $\epsilon$. As such, $\epsilon$ effectively "drops out" of the problem. Strictly speaking, the current theory is only valid for $\epsilon=0$; however, in real spirals, $\epsilon$ is the bifurcation parameter. Thus, this theory is incapable of reproducing the transition.

Coupled with the previous remark, is the fact that numerical work has shown the meandering transition to be a Hopf bifurcation. In this study, we have found that the only unstable mode corresponds to $N=0$, which implies that the mode has a purely real eigenvalue. We surmise that what must be happening is that as $\epsilon$ becomes larger, the eigenvalue of the $N=0$ mode must collide with some other $N=0$ mode (stable 
at $\epsilon=0$ ) becoming complex. If this is true, the complex nature of the bifurcation depends specifically on the coupling between the inner and outer regions which is absent in the limit solved here. One possibility is that this can be studied by going to finite diffusivity ratio (instead of finite $\epsilon$ ) where the core is not decoupled from the far field [29]. Alternatively, one will have to face up to finite $\epsilon$ corrections, a task which may be extremely difficult.

In summary, we have provided the first rigorous calculation of the structure and stability of spiral core in excitable media. We found that the core is generically unstable to a tip-localized mode, as $\epsilon \rightarrow 0$. Further progress in bridging the gap between our asymptotic analysis and the behaviour of spirals as seen in simulation studies must await extensions of our work to either finite $\epsilon$ or finite diffusivity ratio; in both of these cases, the spiral cannot be broken down into outer and core regions since these regions are not of different spatial scales.

\section{Acknowledgements}

We would like to acknowledge the invaluable assistance of Dwight Barkley, as well as useful discussions with A. Karma and A. Winfree. The work of D.A.K. was supported by US Dept. of Energy Grant DE-FG-02-85ER54189, the work of H.L. and W.R. was supported in part by National Science Foundation Grant no. DMR9115413.

\section{References}

[1] E.R. Pinka, Theoretical Ecology: Principles and Application, R.M. May, ed. (Blackwells Scientific, 1981).

[2] A.L. Hodgkin and A.F. Huxley, J. Physiol. (London) 117 (1952) 500.

[3] J.S. Nagumo et al., Proc. IRE 50 (1979a) 509.

[4] R.J. Field, E. Körös and R.J. Noyes, J. Am. Chem. Soc. 94 (1972) 8649.
[5] J.L. Martiel and A. Goldbeter, Biophys. J. 52 (1987) 807.

[6] B.C. Sales, J.E Turner and M.B. Maple, Surface Sci. 114 (1982) 381.

[7] H.L. Swinney and V.I. Krinsky, eds., Workshop on Waves and Patterns in Biological and Chemical Excitable Media, Physica D 49 (1991), deals exclusively with excitable phenomena.

[8] G.S. Skinner and H.L. Swinney, Physica D 48 (1990) 1; T. Plesser, S.C. Muller and B. Hess, J. Phys. Chem. 94 (1990) 7501.

[9] See e.g. D.A. Kessler, J. Koplik and H. Levine, Adv. Phys. 37 (1988) 255.

[10] J.P. Keener, SIAM J. Appl. Math. 46 (1986) 1039.

[11] J.J. Tyson and J.P. Keener, Physica D 32 (1988) 327.

[12] See e.g. V.S. Zykov, in: Spatio-Temporal Organization in Non-equilibrium Systems, S. Muller and Th. Plesser, eds. (Project Verlag, Dortmund).

[13] P. Pelce and J. Sun, Physica D 48 (1991) 353.

[14] J.P. Keener, SIAM J. Appl Math. 52 (1992) 1370.

[15] A. Karma, Phys. Rev. Lett. 66 (1991) 2274.

[16] A.J. Bernoff, Physica D 53 (1991) 125.

[17] A. Karma, Phys. Rev. Lett. 68 (1992) 397.

[18] D.A. Kessler H. Levine and W.N. Reynolds, Phys. Rev. Lett. 68 (1992) 401.

[19] W. Jahnke, W.E. Skaggs and A.T. Winfree, J. Phys. Chem. 93 (1989) 741.

[20] D. Barkley, Physica D 49 (1991) 61.

[21] A. Karma, Phys. Rev. Lett. 65 (1991) 2824.

[22] A. Winfree, Chaos 1 (1991) 303.

[23] D. Barkley, Phys. Rev. Lett. 68 (1992) 2090.

[24] D.A. Kessler, H. Levine and W.N. Reynolds, Phys. Rev. A 46 (1992) 5264.

[25] E. Meron, Pattern formation in excitable media, Phys. Rep. 218 (1992) 1.

[26] D.P. Woodruff, The Solid-liquid Interface (Cambridge Univ. Press, London, 1973).

[27] P.C. Fife, Non-Equilibrium Dynamics in Chemical Systems, C. Vidal and A. Pacault, eds. (Springer, New York, 1984).

[28] W.K. Burton, N. Cabrera and F.C. Frank, Phil. Trans. R. Soc. 243 (1951) 299.

[29] D.A. Kessler and H. Levine, Physica D 49 (1991) 90.

[30] D.A. Kessler, J. Koplik and H. Levine, Phys. Rev. A 31 (1985) 1712.

[31] P. Pelce and J. Sun, Phys. Rev. A 44 (1991) 7906; Physica D 63 (1993) 273.

[32] Harwell Subroutine Library, Computer Science and Systems Division, AERE Harwell, Didcot, Oxon, England.

[33] B.T. Smith, et al., eds., Matrix Eigensystem Routines: EISPACK Guide (Springer, New York, 1976).

[34] D.A. Kessler, H. Levine, and W.N. Reynolds, Europhys. Lett. 19 (1992) 553. 